CRYSTALLOGRAPHIC COMMUNICATIONS

ISSN 2056-9890

Received 1 April 2021

Accepted 6 April 2021

Edited by A. M. Chippindale, University of Reading, England

Keywords: iron(II) complex; thiocyanate complex; high-spin state; trigonal distortion; magnetism; crystal structure.

CCDC reference: 2075540

Supporting information: this article has supporting information at journals.iucr.org/e

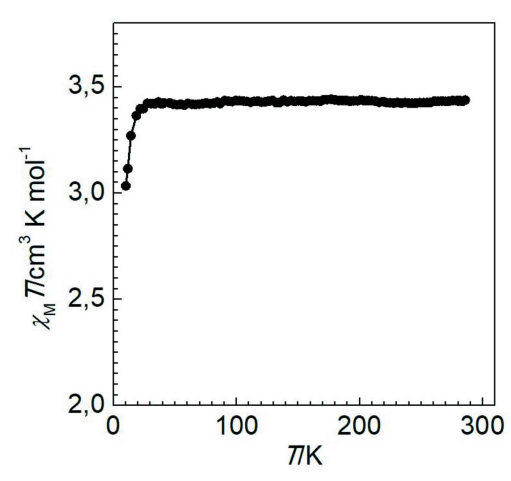

OPEN $\odot$ ACCESS

\section{Crystal structure of $\left(N^{1}, N^{3}\right.$-bis $\{[1-(4-m e t h o x y b e n z-$ yl)-1H-1,2,3-triazol-4-yl]methylidene\}-2,2-dimeth- ylpropane-1,3-diamine)bis(thiocyanato)iron(II)}

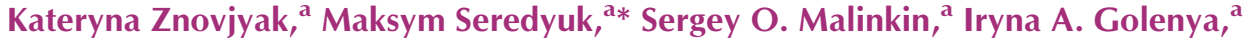 \\ Tatiana Y. Sliva, ${ }^{a}$ Sergiu Shova ${ }^{b}$ and Nurullo U. Mulloev ${ }^{\text {** }}$
}

a Department of Chemistry, Taras Shevchenko National University of Kyiv, Volodymyrska Street 64, Kyiv, 01601, Ukraine, ${ }^{\mathbf{b}}$ Department of Inorganic Polymers, "Petru Poni" Institute of Macromolecular, Chemistry, Romanian Academy of Science, Aleea Grigore Ghica Voda 41-A, lasi, 700487, Romania, and ${ }^{\mathbf{C}}$ The Faculty of Physics, Tajik National University, Rudaki Avenue 17, Dushanbe, 734025, Tajikistan.*Correspondence e-mail: mlseredyuk@gmail.com, voruch@eml.ru

The unit cell of the title compound, $\left[\mathrm{Fe}^{\mathrm{II}}(\mathrm{NCS})_{2}\left(\mathrm{C}_{29} \mathrm{H}_{32} \mathrm{~N}_{8} \mathrm{O}_{2}\right)\right]$, consists of eight charge-neutral complex molecules. In the complex molecule, the tetradentate ligand $N^{1}, N^{3}$-bis $\{[1-(4-m e t h o x y b e n z y l)-1 H-1,2,3-$ triazol-4-yl $]$ methylene $\}-2,2-d i-$ methylpropane-1,3-diamine coordinates to the $\mathrm{Fe}^{\mathrm{II}}$ ion through the $\mathrm{N}$ atoms of the 1,2,3-triazole and aldimine groups. Two thiocyanate anions, coordinated through their $\mathrm{N}$ atoms, complete the coordination sphere of the central $\mathrm{Fe}$ ion. In the crystal, neighbouring molecules are linked through weak $\mathrm{C} \cdots \mathrm{C}, \mathrm{C} \cdots \mathrm{N}$ and $\mathrm{C} \cdots \mathrm{S}$ interactions into a one-dimensional chain running parallel to [010]. The intermolecular contacts were quantified using Hirshfeld surface analysis and two-dimensional fingerprint plots, revealing the relative contributions of the contacts to the crystal packing to be $\mathrm{H} \cdots \mathrm{H}(37.5 \%), \mathrm{H} \cdots \mathrm{C} / \mathrm{C} \cdots \mathrm{H}(24.7 \%)$, $\mathrm{H} \cdots \mathrm{S} / \mathrm{S} \cdots \mathrm{H}(15.7 \%)$ and $\mathrm{H} \cdots \mathrm{N} / \mathrm{N} \cdots \mathrm{H}(11.7 \%)$. The average $\mathrm{Fe}-\mathrm{N}$ bond distance is $2.167 \AA$, indicating the high-spin state of the $\mathrm{Fe}^{\mathrm{II}}$ ion, which does not change upon cooling, as demonstrated by low-temperature magnetic susceptibility measurements.

\section{Chemical context}

$\mathrm{Fe}^{\mathrm{II}}$ complexes based on Schiff bases derived from $N$-substituted 1,2,3-triazole aldehydes represent an interesting class of coordination compounds exhibiting spin-state switching between low- and high-spin states in different temperature regions (Hagiwara et al., 2014, 2016, 2020; Hora \& Hagiwara, 2017). In all of the charge-neutral mononuclear complexes of this kind described so far, the thiocyanate anions occupy the axial position in the coordination sphere and thus are in a trans-configuration (Hagiwara \& Okada, 2016; Hagiwara et al., 2017).

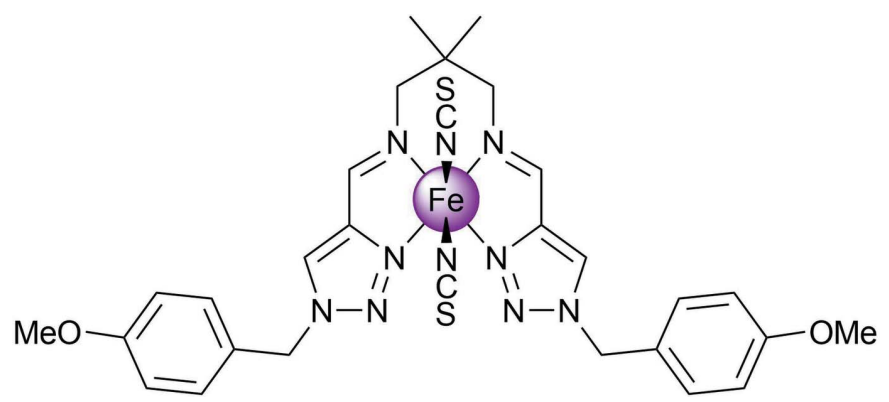

Having ongoing interest in functional $3 d$-metal complexes formed by polydentate ligands (Seredyuk et al., 2006, 2007, 
2011, 2015, 2016; Seredyuk, 2012; Valverde-Muñoz et al., 2020), we report here the synthesis and crystal structure of a new $\mathrm{Fe}^{\mathrm{II}}$ complex based on the tetradentate ligand $N^{1}, N^{3}$ bis \{[1-(4-methoxybenzyl)-1H-1,2,3-triazol-4-yl]methylene\}2,2-dimethylpropane-1,3-diamine with thiocyanate anions arranged around the iron(II) atom in a cis-configuration.

\section{Structural commentary}

The $\mathrm{Fe}^{\mathrm{II}}$ ion of the title complex has a distorted trigonalprismatic $\mathrm{N}_{6}$ coordination environment formed by the four $\mathrm{N}$ atoms of the tetradentate Schiff-base ligand and the two $\mathrm{NCS}^{-}$ counter-ions (Fig. 1). The average bond length, $\langle\mathrm{Fe}-\mathrm{N}\rangle=$ $2.167 \AA$, is typical for high-spin complexes with an $\left[\mathrm{FeN}_{6}\right]$ chromophore (Gütlich \& Goodwin, 2004). The $\mathrm{N}-\mathrm{Fe}-\mathrm{N}$ angle between the cis-aligned thiocyanate $\mathrm{N}$ atoms is $91.6(1)^{\circ}$. The average trigonal distortion parameters, $\Sigma=\Sigma_{1}{ }^{12}\left(\left|90-\varphi_{\mathrm{i}}\right|\right)$, where $\varphi_{\mathrm{i}}$ is the angle $\mathrm{N}-\mathrm{Fe}-\mathrm{N}^{\prime}$ (Drew et al., 1995) and $\Theta=$ $\Sigma_{1}^{24}\left(\left|60-\theta_{\mathrm{i}}\right|\right)$, where $\theta_{\mathrm{i}}$ is the angle generated by the superposition of two opposite faces of an octahedron (Chang et al., 1990), are 127.4 and $481.9^{\circ}$, respectively. The values reveal a great deviation of the coordination environment from an ideal octahedron (where $\Sigma=\Theta=0$ ), and are significantly larger than those of similar $\left[\mathrm{FeN}_{6}\right]$ high-spin trans-complexes (Hagiwara et al., 2017). With the aid of continuous shape measurements (CShM), the shape closest to the Fe-based

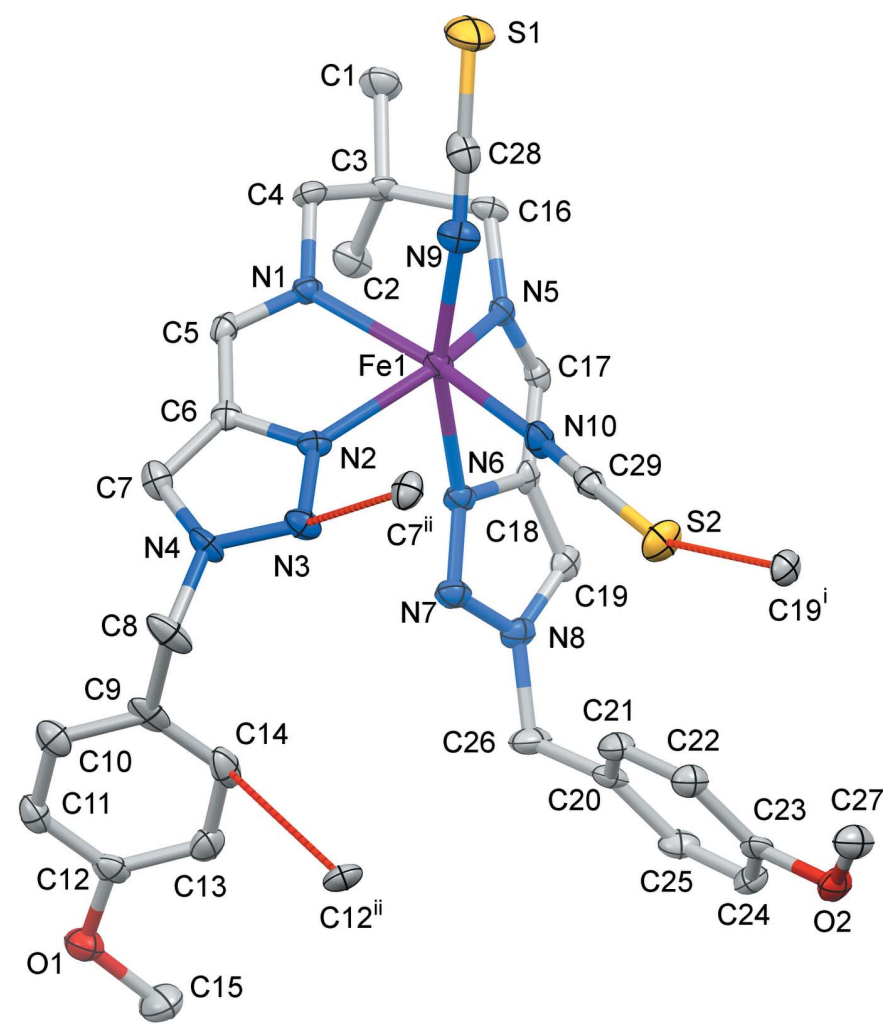

Figure 1

The molecular structure of the title compound with displacement ellipsoids drawn at the $50 \%$ probability level. $\mathrm{H}$ atoms have been omitted for clarity. Weak intermolecular element...element contacts are represented by dashed red lines.
Table 1

Hydrogen-bond geometry $\left(\AA{ }^{\circ}\right)$.

\begin{tabular}{lllll}
\hline$D-\mathrm{H} \cdots A$ & $D-\mathrm{H}$ & $\mathrm{H} \cdots A$ & $D \cdots A$ & $D-\mathrm{H} \cdots A$ \\
\hline $\mathrm{C} 27-\mathrm{H} 27 A \cdots \mathrm{O} 1^{\text {i }}$ & 0.96 & 2.60 & $3.517(4)$ & 161 \\
$\mathrm{C} 20-\mathrm{H} 20 B \cdots \mathrm{O} 2^{\text {ii }}$ & 0.97 & 2.60 & $3.282(4)$ & 127 \\
$\mathrm{C} 19-\mathrm{H} 19 \cdots \mathrm{C} 28^{\text {iii }}$ & 0.93 & 2.75 & $3.574(5)$ & 148 \\
$\mathrm{C} 19-\mathrm{H} 19 \cdots \mathrm{S}$ iii $^{\text {iii }}$ & 0.93 & 2.98 & $3.825(4)$ & 152 \\
$\mathrm{C} 17-\mathrm{H} 17 \cdots \mathrm{N} 10^{\text {iii }}$ & 0.93 & 2.67 & $3.416(4)$ & 138 \\
$\mathrm{C} 17-\mathrm{H} 17 \cdots \mathrm{C} 29^{\mathrm{iii}}$ & 0.93 & 2.85 & $3.685(5)$ & 150 \\
$\mathrm{C} 16-\mathrm{H} 16 A \cdots \mathrm{C} 29^{\text {iii }}$ & 0.97 & 2.73 & $3.667(5)$ & 163 \\
$\mathrm{C} 5-\mathrm{H} 5 \cdots \mathrm{N} 9^{\text {iv }}$ & 0.93 & 2.67 & $3.590(5)$ & 173 \\
$\mathrm{C} 7-\mathrm{H} 7 \cdots \mathrm{N} 10^{\text {iv }}$ & 0.93 & 2.75 & $3.614(5)$ & 156 \\
$\mathrm{C} 7-\mathrm{H} 7 \cdots \mathrm{C} 29^{\text {iv }}$ & 0.93 & 2.49 & $3.400(5)$ & 166 \\
$\mathrm{C} 7-\mathrm{H} 7 \cdots 2^{\text {iv }}$ & 0.93 & 2.99 & $3.752(5)$ & 140 \\
\hline
\end{tabular}

Symmetry codes: (i) $\quad x+\frac{1}{2}, y-1,-z+\frac{1}{2} ; \quad$ (ii) $\quad-x+1, y+\frac{1}{2},-z+\frac{1}{2}$; $-x+1,-y+1,-z+1$; (iv) $-x+\frac{1}{2}, y+\frac{1}{2}, z$.

coordination polyhedron and its distortion can be determined numerically (Kershaw Cook et al., 2015). The calculated CShM value relative to ideal $O_{h}$ symmetry is 4.269 , and 5.671 relative to ideal $D_{3 h}$ trigonal-prismatic symmetry. Hence, the coordination polyhedron is closer to the former geometry, but is appreciably distorted, as indicated by the calculated value (for an ideal polyhedron $\mathrm{CShM}=0$ ). The volume of the $\left[\mathrm{FeN}_{6}\right]$ coordination polyhedron is $12.50 \AA^{3}$.

\section{Supramolecular features}

In the crystal, neighbouring complex molecules form onedimensional supramolecular chains propagating parallel to [010] through weak contacts $\left[\mathrm{S} 2 \cdots \mathrm{C} 19^{\mathrm{i}}=3.271(3) \AA\right.$, $\mathrm{N} 3 \cdots \mathrm{C} 7^{\mathrm{ii}}=3.161(3) \AA$ and $\mathrm{C} 14 \cdots \mathrm{C} 12^{\mathrm{ii}}=3.320$ (3) $\AA$;

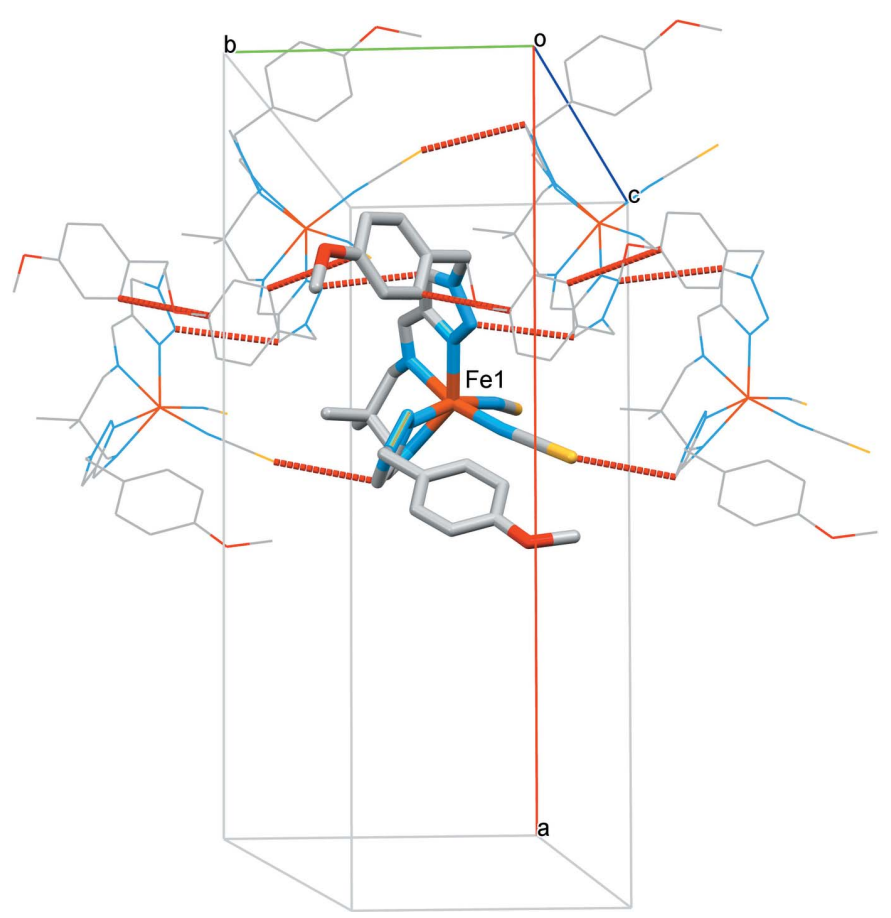

Figure 2

The packing of molecules into one-dimensional chains running parallel to [010] held together by weak C...C/N/S bonding. 

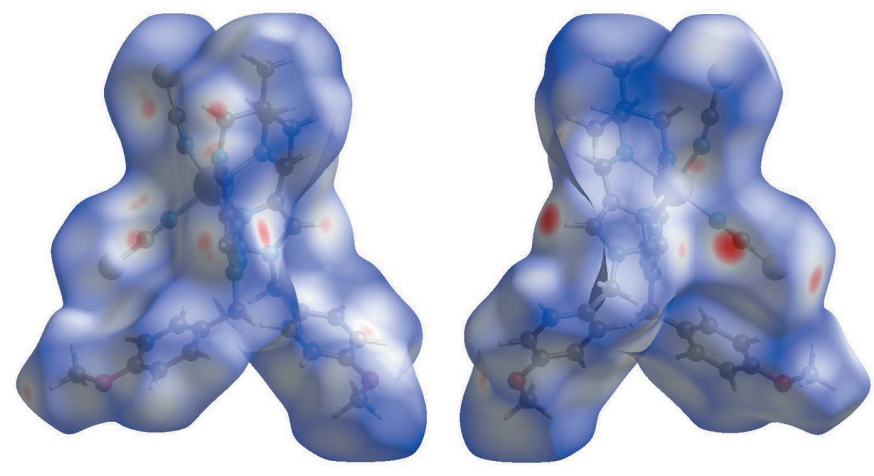

Figure 3

Two projections of $d_{\text {norm }}$ mapped on Hirshfeld surfaces, showing the interactions between molecules. Red areas represent regions where contacts are shorter than the sum of the van der Waals radii, blue areas represent regions where contacts are larger than the sum of van der Waals radii, and white areas are regions where contacts are close to the sum of van der Waals radii.

symmetry codes: (i) $x,-1+y, z$; (ii) $\left.\frac{1}{2}-x,-\frac{1}{2}+y, z\right]$ (Fig. 2). Weak $\mathrm{C}-\mathrm{H} \cdots X$ hydrogen bonds (Table 1 ) link the chains into a three-dimensional network. No strong hydrogen-bonding or stacking interactions are observed between the complex molecules in the crystal structure.

\section{Hirshfeld surface and 2D fingerprint plots}

Hirshfeld surface analysis was performed and the associated two-dimensional fingerprint plots were generated using Crystal Explorer (Turner et al., 2017), with a standard resolution of the three-dimensional $d_{\text {norm }}$ surfaces plotted over a fixed colour scale of -0.3171 (red) to 1.6637 (blue) a.u. (Fig. 3). The pale-red spots symbolize short contacts and negative $d_{\text {norm }}$ values on the surface correspond to the interactions described above. The Hirshfeld surfaces mapped over $d_{\text {norm }}$ are shown for the $\mathrm{H} \cdots \mathrm{H}, \mathrm{H} \cdots \mathrm{C} / \mathrm{C} \cdots \mathrm{H}, \mathrm{H} \cdots \mathrm{S} / \mathrm{S} \cdots \mathrm{H}$, and $\mathrm{H} \cdots \mathrm{N} / \mathrm{N} \cdots \mathrm{H}$ contacts, and the two-dimensional fingerprint plots are presented in Fig. 4, associated with their relative contributions to the Hirshfeld surface. At $37.5 \%$, the largest contribution to the overall crystal packing is from $\mathrm{H} \cdots \mathrm{H}$ interactions, which are located in the middle region of the fingerprint plot. $\mathrm{H} \cdots \mathrm{C} / \mathrm{C} \cdots \mathrm{H}$ contacts contribute $24.7 \%$, and the $\mathrm{H} \cdots \mathrm{S} / \mathrm{S} \cdots \mathrm{H}$ contacts contribute $15.7 \%$ to the Hirshfeld surface, both resulting in a pair of characteristic wings. The $\mathrm{H} \cdots \mathrm{N} / \mathrm{N} \cdots \mathrm{H}$ contacts, represented by a pair of sharp spikes in the fingerprint plot, make a $11.7 \%$ contribution to the Hirshfeld surface.

\section{Magnetic properties}

Variable-temperature magnetic susceptibility measurements were performed on single crystals $(10 \mathrm{mg})$ of the title compound using a Quantum Design MPMS2 superconducting quantum interference device (SQUID) susceptometer operating at $1 \mathrm{~T}$ in the temperature range $10-400 \mathrm{~K}$. Experimental susceptibilities were corrected for the diamagnetism of the holder (gelatine capsule) and of the constituent atoms by the (a)
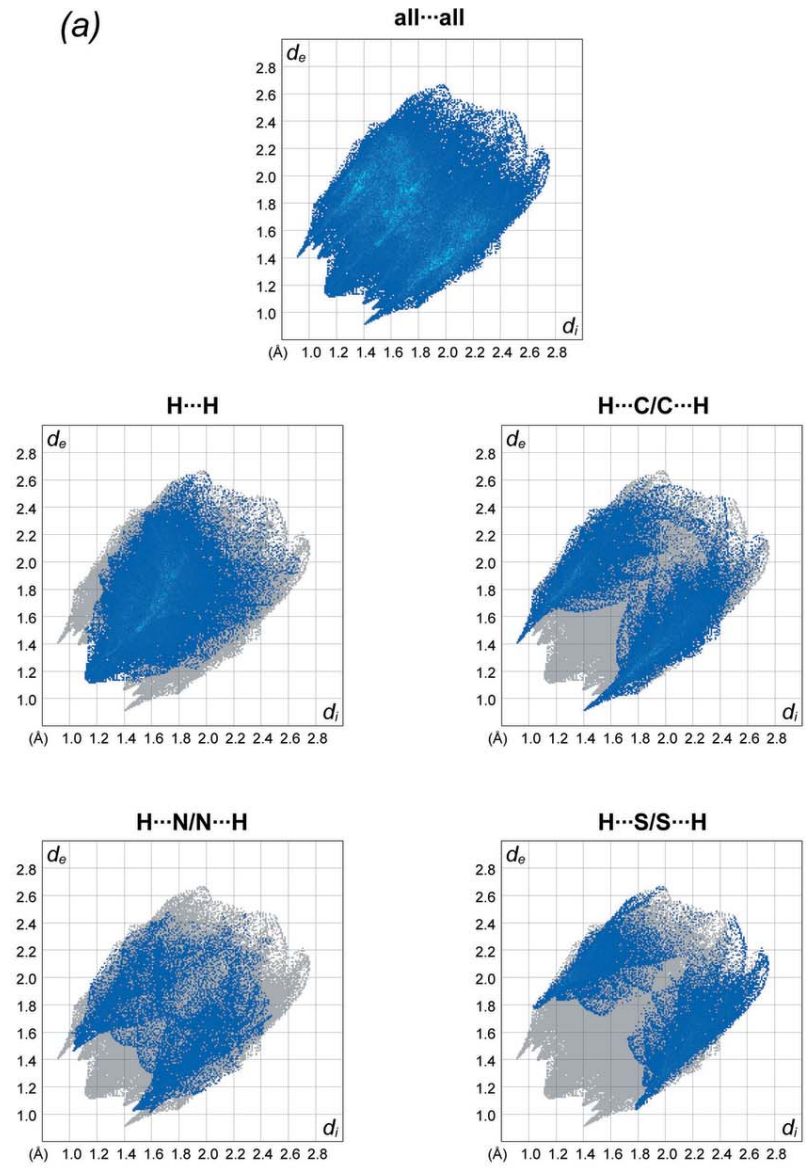

(b)
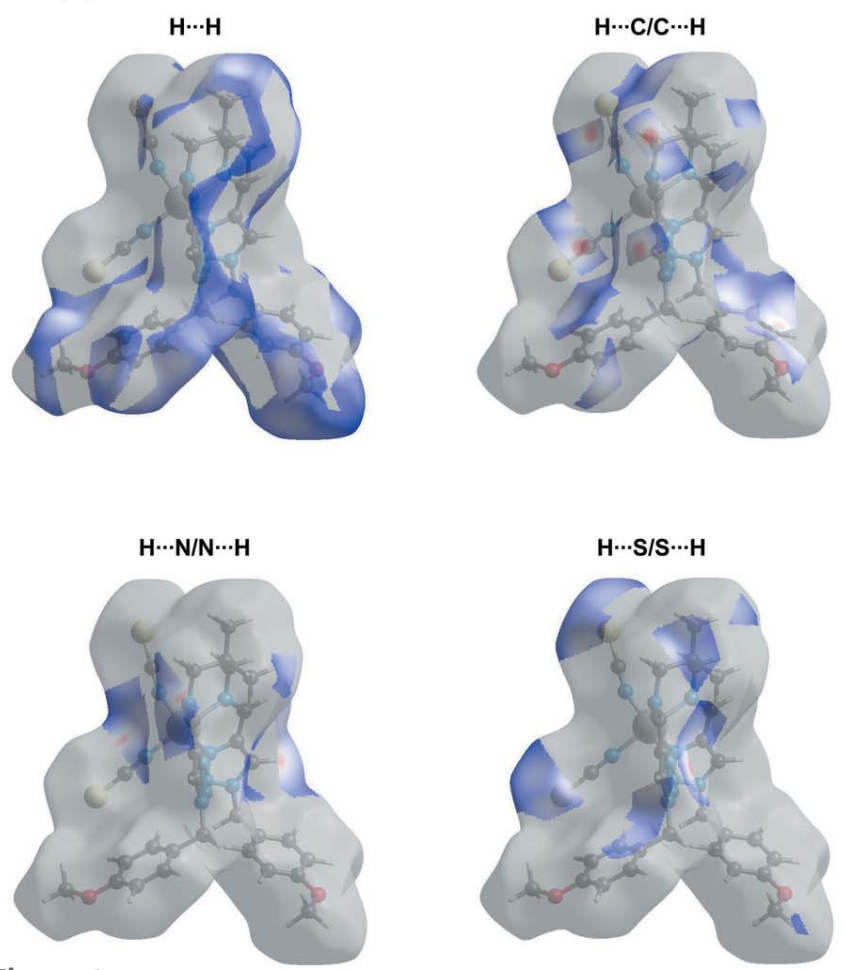

Figure 4

(a) The overall two-dimensional fingerprint plot and those delineated into specified interactions. (b) Hirshfeld surface representations with the function $d_{\text {norm }}$ plotted onto the surface for the different interactions. 
Table 2

Comparison of the distortion parameters $\left(\AA,{ }^{\circ}\right)$ for indicated $\mathrm{Fe}^{\mathrm{II}}$ complexes.

\begin{tabular}{lllll}
\hline & $<\mathrm{Fe}-\mathrm{N}\rangle$ & $\Sigma$ & $\Theta$ & $\operatorname{CShM}\left(D_{3 h}\right)$ \\
\hline Title compound & 2.167 & 127.4 & 481.9 & 5.671 \\
CUWQAP & 2.186 & 149.38 & 453.2 & 4.008 \\
CABLOH & 1.899 & 725.74 & 178.16 & 0.525 \\
BUNSAF & 2.218 & 703.65 & 201.07 & 1.887 \\
OWIHAE & 2.202 & 894.48 & 206.57 & 0.602 \\
OTANOO $^{a}$ & 2.191 & 697.3 & 183.24 & 1.098 \\
\hline
\end{tabular}

Note: (a) Parameters averaged over five independent complex cations.

application of Pascal's constants. The magnetic behaviour of the compound is shown in Fig. 5 in the form of $\chi_{\mathrm{M}} T$ versus $T$ ( $\chi_{\mathrm{M}}$ is the molar magnetic susceptibility and $T$ is the temperature). At $300 \mathrm{~K}$, the $\chi_{\mathrm{M}} T$ value is close to $3.40 \mathrm{~cm}^{3} \mathrm{~K} \mathrm{~mol}^{-1}$, and on cooling the value remains constant down to $30 \mathrm{~K}$. The decrease in $\chi_{\mathrm{M}} T$ below $30 \mathrm{~K}$ is attributed to the zero-field splitting of the high-spin $(S=2) \mathrm{Fe}^{\mathrm{II}}$ centres (Kahn, 1993), which corroborates well with the observed long average $\mathrm{Fe}-\mathrm{N}$ bond length and the large geometric distortion of the coordination polyhedron of the central $\mathrm{Fe}^{\mathrm{II}}$ ion.

\section{Database survey}

A search of the Cambridge Structural Database (CSD, online) reveals five similar $\mathrm{Fe}^{\mathrm{II}}$ thiocyanate complexes: derivatives of 1,3-diamine and $N$-substituted 1,2,3-triazole aldehydes: DURXEV, ADAQUU, ADAREF and solvatomorphs ADAROP and ADARUV (Hagiwara et al., 2017; Hagiwara \& Okada, 2016). These complexes show hysteretic spin crossover with variation of the $\mathrm{Fe}-\mathrm{N}$ distances in the range 1.931$1.959 \AA$ for the low-spin state and 2.154-2.169 $\AA$ for the highspin state of the $\mathrm{Fe}^{\mathrm{II}}$ ions. The reported pseudo-trigonalprismatic complexes with an $\left[\mathrm{FeN}_{6}\right]$ chromophore are formed by structurally hindered rigid hexadentate ligands favouring a

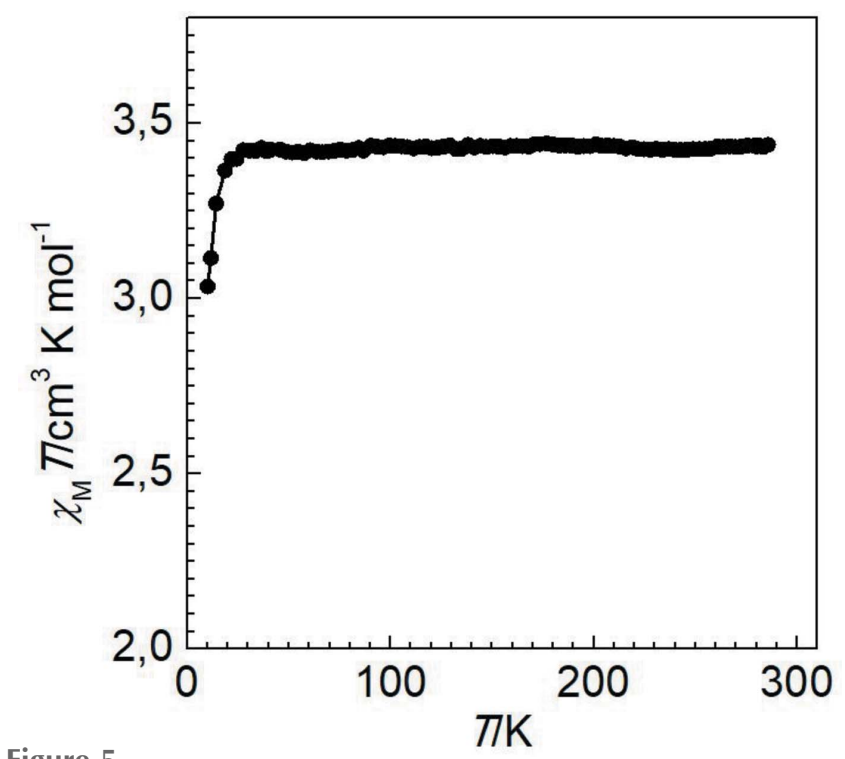

Figure 5

$\chi_{\mathrm{M}} T$ versus $T$ plot for the title compound.
Table 3

Experimental details.

\begin{tabular}{|c|c|}
\hline \multicolumn{2}{|l|}{ Crystal data } \\
\hline Chemical formula & {$\left[\mathrm{Fe}(\mathrm{NCS})_{2}\left(\mathrm{C}_{27} \mathrm{H}_{32} \mathrm{~N}_{8} \mathrm{O}_{2}\right)\right]$} \\
\hline$M_{\mathrm{r}}$ & 672.61 \\
\hline Crystal system, space group & Orthorhombic, $\mathrm{Pbca}$ \\
\hline Temperature $(\mathrm{K})$ & 99 \\
\hline$a, b, c(\AA)$ & $\begin{array}{l}22.8809(15), 9.0485(4), \\
\quad 31.2662(18)\end{array}$ \\
\hline$V\left(\AA^{3}\right)$ & $6473.3(6)$ \\
\hline$Z$ & 8 \\
\hline Radiation type & Мo $K \alpha$ \\
\hline$\mu\left(\mathrm{mm}^{-1}\right)$ & 0.64 \\
\hline Crystal size $(\mathrm{mm})$ & $0.3 \times 0.2 \times 0.05$ \\
\hline \multicolumn{2}{|l|}{ Data collection } \\
\hline Diffractometer & $\begin{array}{l}\text { Rigaku Oxford Diffraction } \\
\text { Xcalibur, Eos }\end{array}$ \\
\hline Absorption correction & $\begin{array}{l}\text { Multi-scan (CrysAlis PRO; Rigaku } \\
\text { OD, 2015) }\end{array}$ \\
\hline$T_{\min }, T_{\max }$ & $0.983,1.000$ \\
\hline $\begin{array}{l}\text { No. of measured, independent and } \\
\text { observed }[I>2 \sigma(I)] \text { reflections }\end{array}$ & $14323,5718,4331$ \\
\hline$R_{\text {int }}$ & 0.062 \\
\hline \multicolumn{2}{|l|}{ Refinement } \\
\hline$R\left[F^{2}>2 \sigma\left(F^{2}\right)\right], w R\left(F^{2}\right), S$ & $0.059,0.111,1.10$ \\
\hline No. of reflections & 5718 \\
\hline No. of parameters & 401 \\
\hline $\mathrm{H}$-atom treatment & H-atom parameters constrained \\
\hline$\Delta \rho_{\max }, \Delta \rho_{\min }\left(\mathrm{e} \AA^{-3}\right)$ & $0.45,-0.35$ \\
\hline
\end{tabular}

Computer programs: CrysAlis PRO (Rigaku OD, 2015), SIR2008 (Burla et al., 2007), SHELXL2018/3 (Sheldrick, 2015) and OLEX2 (Dolomanov et al., 2009).

trigonal geometry of the central $\mathrm{Fe}^{\mathrm{II}}$ ion: $\mathrm{CABLOH}$ (Voloshin et al., 2001), BUNSAF (El Hajj et al., 2009), OWIHAE (Seredyuk et al., 2011), OTANOO (Stock et al., 2016). The complex CUWQAP, recently reported by us (Znovjyak et al., 2020), has a similar strongly distorted coordination environment of the central $\mathrm{Fe}^{\mathrm{II}}$ ion. Table 2 collates the distortion parameters $\Sigma, \Theta$ and CShM for the pseudo-trigonal-prismatic complexes mentioned above.

\section{Synthesis and crystallization}

The ligand of the title compound was obtained in situ by condensation of 2,2-dimethyl-1,3-propanediamine $(24 \mu \mathrm{L}$, $0.20 \mathrm{mmol})$ with 1-(4-methoxybenzyl)-1H-1,2,3-triazole-4carbaldehyde ( $92 \mathrm{mg}, 0.45 \mathrm{mmol}$ ) by boiling in methanol for $5 \mathrm{~min}$ and was subsequently reacted with $\left[\mathrm{Fe}(\mathrm{py})_{4}(\mathrm{NCS})_{2}\right]$ (100 mg, $0.20 \mathrm{mmol}$ ) and ascorbic acid (11 mg, $0.06 \mathrm{mmol})$ dissolved in a minimum of boiling methanol. The yellow solution formed was slowly cooled to ambient temperature. Yellow-orange crystals then precipitated and were filtered off. Elemental analysis calculated (\%) for $\mathrm{C}_{29} \mathrm{H}_{32} \mathrm{FeN}_{10} \mathrm{O}_{2} \mathrm{~S}_{2}$ : C, 51.79; H, 4.80; N, 20.82; S, 9.53. Found: C, 52.02; H, 4.68; N, 20.77; S, 9.40. IR $v\left(\mathrm{~cm}^{-1}, \mathrm{KBr}\right): 1614(\mathrm{C}=\mathrm{N}), 2070,2118$ (NCS).

\section{Refinement}

Crystal data, data collection and structure refinement details are summarized in Table 3. $\mathrm{H}$ atoms were positioned geom- 
etrically $(\mathrm{C}-\mathrm{H}=0.93-0.97 \AA)$ and refined as riding with $U_{\text {iso }}(\mathrm{H})=1.2 U_{\text {eq }}(\mathrm{C})$ or $1.5 U_{\text {eq }}(\mathrm{C}$-methyl $)$.

\section{Acknowledgements}

Authors contributions are as follows: Conceptualization, NUM and MS; methodology, KZ; formal analysis, NUM; synthesis, SOM; magnetic measurements, IAG; single crystal measurements, SS; writing (original draft), NUM and MS; writing (review and editing of the manuscript), NUM, MS, KZ, SOM, IAG, TYS and SS; visualization, TYS; funding acquisition, KZ.

\section{Funding information}

Funding for this research was provided by: H2020 Marie Skłodowska-Curie Actions (grant No. 734322).

\section{References}

Burla, M. C., Caliandro, R., Camalli, M., Carrozzini, B., Cascarano, G. L., De Caro, L., Giacovazzo, C., Polidori, G., Siliqi, D. \& Spagna, R. (2007). J. Appl. Cryst. 40, 609-613.

Chang, H. R., McCusker, J. K., Toftlund, H., Wilson, S. R., Trautwein, A. X., Winkler, H. \& Hendrickson, D. N. (1990). J. Am. Chem. Soc. 112, 6814-6827.

Dolomanov, O. V., Bourhis, L. J., Gildea, R. J., Howard, J. A. K. \& Puschmann, H. (2009). J. Appl. Cryst. 42, 339-341.

Drew, M. G. B., Harding, C. J., McKee, V., Morgan, G. G. \& Nelson, J. (1995). J. Chem. Soc. Chem. Commun. pp. 1035-1038.

El Hajj, F., Sebki, G., Patinec, V., Marchivie, M., Triki, S., Handel, H., Yefsah, S., Tripier, R., Gómez-García, C. J. \& Coronado, E. (2009). Inorg. Chem. 48, 10416-10423.

Gütlich, P. \& Goodwin, H. A. (2004). Top. Curr. Chem. 233, 1-47.

Hagiwara, H., Masuda, T., Ohno, T., Suzuki, M., Udagawa, T. \& Murai, K.-I. (2017). Cryst. Growth Des. 17, 6006-6019.

Hagiwara, H., Minoura, R., Okada, S. \& Sunatsuki, Y. (2014). Chem. Lett. 43, 950-952.

Hagiwara, H., Minoura, R., Udagawa, T., Mibu, K. \& Okabayashi, J. (2020). Inorg. Chem. 59, 9866-9880.
Hagiwara, H. \& Okada, S. (2016). Chem. Commun. 52, 815-818.

Hagiwara, H., Tanaka, T. \& Hora, S. (2016). Dalton Trans. 45, 1713217140.

Hora, S. \& Hagiwara, H. (2017). Inorganics, 5, 49.

Kahn, O. (1993). Molecular Magnetism. New York: Wiley-VCH.

Kershaw Cook, L. J., Mohammed, R., Sherborne, G., Roberts, T. D., Alvarez, S. \& Halcrow, M. A. (2015). Coord. Chem. Rev. 289-290, $2-12$.

Rigaku OD (2015). CrysAlis PRO. Rigaku Oxford Diffraction, Yarnton, England.

Seredyuk, M. (2012). Inorg. Chim. Acta, 380, 65-71.

Seredyuk, M., Gaspar, A. B., Ksenofontov, V., Reiman, S., Galyametdinov, Y., Haase, W., Rentschler, E. \& Gütlich, P. (2006). Hyperfine Interact. 166, 385-390.

Seredyuk, M., Gaspar, A. B., Kusz, J. \& Gütlich, P. (2011). Z. Anorg. Allg. Chem. 637, 965-976.

Seredyuk, M., Haukka, M., Fritsky, I. O., Kozłowski, H., Krämer, R., Pavlenko, V. A. \& Gütlich, P. (2007). Dalton Trans. pp. 31833194.

Seredyuk, M., Piñeiro-López, L., Muñoz, M. C., Martínez-Casado, F. J., Molnár, G., Rodriguez-Velamazán, J. A., Bousseksou, A. \& Real, J. A. (2015). Inorg. Chem. 54, 7424-7432.

Seredyuk, M., Znovjyak, K., Muñoz, M. C., Galyametdinov, Y., Fritsky, I. O. \& Real, J. A. (2016). RSC Adv. 6, 39627-39635.

Sheldrick, G. M. (2015). Acta Cryst. C71, 3-8.

Stock, P., Deck, E., Hohnstein, S., Korzekwa, J., Meyer, K., Heinemann, F. W., Breher, F. \& Hörner, G. (2016). Inorg. Chem. 55, 5254-5265.

Turner, M. J., McKinnon, J. J., Wolff, S. K., Grimwood, D. J., Spackman, P. R., Jayatilaka, D. \& Spackman, M. A. (2017). CrystalExplorer 17. University of Western Australia. http:// hirshfeldsurface.net.

Valverde-Muñoz, F., Seredyuk, M., Muñoz, M. C., Molnár, G., Bibik, Y. S. \& Real, J. A. (2020). Angew. Chem. Int. Ed. 59, 1863218638.

Voloshin, Y. Z., Varzatskii, O. A., Stash, A. I., Belsky, V. K., Bubnov, Y. N., Vorontsov, I. I., Potekhin, K. A., Antipin, M. Y. \& Polshin, E. V. (2001). Polyhedron, 20, 2721-2733.

Znovjyak, K., Seredyuk, M., Malinkin, S. O., Shova, S. \& Soliev, L. (2020). Acta Cryst. E76, 1661-1664. 


\section{supporting information}

Acta Cryst. (2021). E77, 495-499 [https://doi.org/10.1107/S2056989021003662]

Crystal structure of $\left(N^{1}, N^{3}\right.$-bis $\{[1-(4-m e t h o x y b e n z y l)-1 H-1,2,3-$ triazol-4yl]methylidene\}-2,2-dimethylpropane-1,3-diamine)bis(thiocyanato)iron(II)

Kateryna Znovjyak, Maksym Seredyuk, Sergey O. Malinkin, Iryna A. Golenya, Tatiana Y. Sliva, Sergiu Shova and Nurullo U. Mulloev

Computing details

Data collection: CrysAlis PRO (Rigaku OD, 2015); cell refinement: CrysAlis PRO (Rigaku OD, 2015); data reduction: CrysAlis PRO (Rigaku OD, 2015); program(s) used to solve structure: SIR2008 (Burla et al., 2007); program(s) used to refine structure: SHELXL2018/3 (Sheldrick, 2015); molecular graphics: OLEX2 (Dolomanov et al., 2009); software used to prepare material for publication: OLEX2 (Dolomanov et al., 2009).

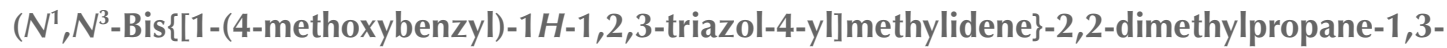
diamine)bis(thiocyanato)iron(II)

Crystal data

$\left[\mathrm{Fe}(\mathrm{NCS})_{2}\left(\mathrm{C}_{27} \mathrm{H}_{32} \mathrm{~N}_{8} \mathrm{O}_{2}\right)\right]$

$M_{r}=672.61$

Orthorhombic, $\mathrm{Pbca}$

$a=22.8809$ (15) $\AA$

$b=9.0485(4) \AA$

$c=31.2662(18) \AA$

$V=6473.3(6) \AA^{3}$

$Z=8$

$F(000)=2800$

Data collection

Rigaku Oxford Diffraction Xcalibur, Eos diffractometer

Detector resolution: 8.0797 pixels $\mathrm{mm}^{-1}$

$\omega$ scans

Absorption correction: multi-scan

(CrysAlisPro; Rigaku OD, 2015)

$T_{\min }=0.983, T_{\max }=1.000$

14323 measured reflections

Refinement

Refinement on $F^{2}$

Least-squares matrix: full

$R\left[F^{2}>2 \sigma\left(F^{2}\right)\right]=0.059$

$w R\left(F^{2}\right)=0.111$

$S=1.10$

5718 reflections
$D_{\mathrm{x}}=1.380 \mathrm{Mg} \mathrm{m}^{-3}$

Mo $K \alpha$ radiation, $\lambda=0.71073 \AA$

Cell parameters from 3410 reflections

$\theta=2.2-26.9^{\circ}$

$\mu=0.64 \mathrm{~mm}^{-1}$

$T=99 \mathrm{~K}$

Plate, clear dark red

$0.3 \times 0.2 \times 0.05 \mathrm{~mm}$

5718 independent reflections

4331 reflections with $I>2 \sigma(I)$

$R_{\text {int }}=0.062$

$\theta_{\text {max }}=25.0^{\circ}, \theta_{\min }=1.8^{\circ}$

$h=-10 \rightarrow 27$

$k=-10 \rightarrow 10$

$l=-35 \rightarrow 37$

401 parameters

0 restraints

Hydrogen site location: inferred from neighbouring sites

$\mathrm{H}$-atom parameters constrained 
$w=1 /\left[\sigma^{2}\left(F_{\mathrm{o}}^{2}\right)+(0.0332 P)^{2}\right]$

where $P=\left(F_{\mathrm{o}}^{2}+2 F_{\mathrm{c}}^{2}\right) / 3$

$(\Delta / \sigma)_{\max }<0.001$

$$
\Delta \rho_{\max }=0.45 \text { e } \AA^{-3}
$$

$\Delta \rho_{\min }=-0.35$ e $\AA^{-3}$

\section{Special details}

Geometry. All esds (except the esd in the dihedral angle between two 1.s. planes) are estimated using the full covariance matrix. The cell esds are taken into account individually in the estimation of esds in distances, angles and torsion angles; correlations between esds in cell parameters are only used when they are defined by crystal symmetry. An approximate (isotropic) treatment of cell esds is used for estimating esds involving l.s. planes.

Fractional atomic coordinates and isotropic or equivalent isotropic displacement parameters $\left(\AA^{2}\right)$

\begin{tabular}{|c|c|c|c|c|}
\hline & $x$ & $y$ & $z$ & $U_{\text {iso }} * / U_{\text {eq }}$ \\
\hline Fe1 & $0.36927(2)$ & $0.44257(5)$ & $0.48750(2)$ & $0.01653(14)$ \\
\hline $\mathrm{S} 1$ & $0.35511(5)$ & 0.27805 (13) & $0.63189(3)$ & $0.0447(3)$ \\
\hline $\mathrm{S} 2$ & $0.45693(4)$ & $0.01869(10)$ & $0.41509(3)$ & 0.0291 \\
\hline $\mathrm{O} 1$ & $0.21074(11)$ & $0.7698(3)$ & $0.22792(7)$ & $0.0291(6)$ \\
\hline $\mathrm{O} 2$ & $0.59076(10)$ & $0.1143(2)$ & $0.27020(7)$ & $0.0248(6)$ \\
\hline N1 & $0.30621(12)$ & $0.6015(3)$ & $0.51744(8)$ & $0.0178(7)$ \\
\hline N2 & $0.29144(12)$ & 0.4260 & $0.44994(8)$ & $0.0170(7)$ \\
\hline N3 & $0.27703(12)$ & $0.3551(3)$ & $0.41465(9)$ & $0.0214(7)$ \\
\hline N4 & $0.22437(12)$ & $0.4080(3)$ & $0.40340(9)$ & $0.0206(7)$ \\
\hline N5 & $0.42995(12)$ & 0.6039 & $0.51301(9)$ & $0.0164(6)$ \\
\hline N6 & $0.40524(12)$ & $0.5724(3)$ & $0.43049(9)$ & $0.0187(7)$ \\
\hline N7 & $0.40240(13)$ & $0.5591(3)$ & $0.38880(9)$ & $0.0219(7)$ \\
\hline N8 & $0.45248(13)$ & $0.6174(3)$ & $0.37344(9)$ & $0.0211(7)$ \\
\hline N9 & $0.36115(13)$ & 0.3225 & $0.54366(10)$ & $0.0257(7)$ \\
\hline N10 & $0.41108(12)$ & 0.2661 & $0.45691(9)$ & $0.0227(7)$ \\
\hline $\mathrm{C} 1$ & $0.37528(17)$ & $0.8159(4)$ & $0.60960(11)$ & $0.0310(10)$ \\
\hline H1A & 0.375354 & 0.733805 & 0.629056 & $0.047^{*}$ \\
\hline H1B & 0.342017 & 0.877495 & 0.615288 & $0.047 *$ \\
\hline $\mathrm{H} 1 \mathrm{C}$ & 0.410447 & 0.872295 & 0.613305 & $0.047^{*}$ \\
\hline C3 & $0.37212(15)$ & $0.7588(4)$ & $0.56379(11)$ & $0.0193(8)$ \\
\hline C4 & $0.31474(15)$ & $0.6730(4)$ & $0.55938(10)$ & $0.0227(9)$ \\
\hline $\mathrm{H} 4 \mathrm{~A}$ & 0.313404 & 0.597677 & 0.581427 & $0.027 *$ \\
\hline H4B & 0.282487 & 0.740361 & 0.564420 & $0.027^{*}$ \\
\hline C16 & $0.42617(15)$ & $0.6583(4)$ & $0.55718(10)$ & $0.0196(8)$ \\
\hline H16A & 0.461308 & 0.713397 & 0.564006 & $0.024 *$ \\
\hline H16B & 0.423858 & 0.574790 & 0.576577 & $0.024 *$ \\
\hline $\mathrm{C} 5$ & $0.25706(15)$ & $0.6127(4)$ & $0.49871(10)$ & $0.0185(8)$ \\
\hline H5 & 0.227835 & 0.674579 & 0.509020 & $0.022 *$ \\
\hline C6 & $0.24881(15)$ & $0.5244(3)$ & $0.46060(11)$ & $0.0166(8)$ \\
\hline $\mathrm{C} 7$ & $0.20567(15)$ & $0.5129(4)$ & $0.43064(11)$ & $0.0233(9)$ \\
\hline $\mathrm{H} 7$ & 0.170935 & 0.566109 & 0.429329 & $0.028 *$ \\
\hline $\mathrm{C} 8$ & 0.19663 (18) & $0.3560(4)$ & $0.36374(11)$ & $0.0319(10)$ \\
\hline H8A & 0.155587 & 0.336456 & 0.369061 & $0.038^{*}$ \\
\hline H8B & 0.214826 & 0.264241 & 0.354803 & $0.038 *$ \\
\hline C9 & $0.20226(17)$ & $0.4690(4)$ & $0.32836(11)$ & $0.0252(9)$ \\
\hline $\mathrm{C} 10$ & $0.15496(16)$ & $0.5551(4)$ & $0.31594(11)$ & $0.0255(9)$ \\
\hline
\end{tabular}




\begin{tabular}{|c|c|c|c|c|}
\hline H10 & 0.119558 & 0.544958 & 0.330250 & $0.031^{*}$ \\
\hline $\mathrm{C} 11$ & $0.15932(16)$ & 0.6545 (4) & $0.28308(11)$ & $0.0243(9)$ \\
\hline H11 & 0.127045 & 0.711095 & 0.275409 & $0.029^{*}$ \\
\hline $\mathrm{C} 12$ & $0.21154(16)$ & $0.6713(4)$ & $0.26122(11)$ & $0.0227(9)$ \\
\hline $\mathrm{C} 13$ & $0.26012(16)$ & 0.5918 (4) & $0.27391(11)$ & $0.0279(9)$ \\
\hline H13 & 0.295853 & 0.605572 & 0.260294 & $0.033^{*}$ \\
\hline C14 & $0.25489(16)$ & 0.4906 (4) & $0.30743(12)$ & $0.0285(9)$ \\
\hline H14 & 0.287484 & 0.436628 & 0.315875 & $0.034 *$ \\
\hline C15 & $0.26393(17)$ & 0.7909 (5) & $0.20445(12)$ & $0.0402(11)$ \\
\hline $\mathrm{H} 15 \mathrm{~A}$ & 0.257419 & 0.860635 & 0.181795 & $0.060 *$ \\
\hline H15B & 0.276332 & 0.698306 & 0.192538 & $0.060 *$ \\
\hline $\mathrm{H} 15 \mathrm{C}$ & 0.293636 & 0.827953 & 0.223293 & $0.060^{*}$ \\
\hline $\mathrm{C} 17$ & $0.46360(15)$ & $0.6705(4)$ & $0.48662(11)$ & $0.0185(8)$ \\
\hline H17 & 0.491715 & 0.737278 & 0.496004 & $0.022 *$ \\
\hline C18 & $0.45624(14)$ & $0.6381(3)$ & $0.44164(11)$ & $0.0159(8)$ \\
\hline $\mathrm{C} 19$ & $0.48706(16)$ & $0.6670(3)$ & $0.40516(11)$ & $0.0201(8)$ \\
\hline H19 & 0.523657 & 0.710946 & 0.402782 & $0.024 *$ \\
\hline $\mathrm{C} 20$ & $0.46345(17)$ & 0.6144 (4) & $0.32700(11)$ & $0.0291(10)$ \\
\hline $\mathrm{H} 20 \mathrm{~A}$ & 0.485754 & 0.701335 & 0.319094 & $0.035^{*}$ \\
\hline $\mathrm{H} 20 \mathrm{~B}$ & 0.426354 & 0.618584 & 0.312035 & $0.035^{*}$ \\
\hline $\mathrm{C} 21$ & $0.49624(15)$ & $0.4783(4)$ & $0.31281(10)$ & $0.0196(8)$ \\
\hline $\mathrm{C} 22$ & $0.54372(15)$ & 0.4919 (4) & $0.28529(10)$ & $0.0231(9)$ \\
\hline $\mathrm{H} 22$ & 0.555641 & 0.585120 & 0.276344 & $0.028 *$ \\
\hline $\mathrm{C} 23$ & $0.57315(15)$ & 0.3689 (4) & $0.27121(11)$ & $0.0230(9)$ \\
\hline $\mathrm{H} 23$ & 0.604410 & 0.379555 & 0.252458 & $0.028^{*}$ \\
\hline $\mathrm{C} 24$ & $0.55672(15)$ & $0.2287(4)$ & $0.28470(10)$ & $0.0190(8)$ \\
\hline $\mathrm{C} 25$ & $0.50976(15)$ & $0.2136(4)$ & $0.31199(11)$ & $0.0219(8)$ \\
\hline $\mathrm{H} 25$ & 0.498300 & 0.120252 & 0.321203 & $0.026^{*}$ \\
\hline $\mathrm{C} 26$ & $0.47962(16)$ & $0.3382(4)$ & $0.32569(11)$ & $0.0232(9)$ \\
\hline H26 & 0.447708 & 0.327227 & 0.343831 & $0.028^{*}$ \\
\hline $\mathrm{C} 27$ & $0.58123(16)$ & $-0.0289(4)$ & $0.28871(11)$ & $0.0283(9)$ \\
\hline $\mathrm{H} 27 \mathrm{~A}$ & 0.611071 & -0.095612 & 0.279086 & $0.042 *$ \\
\hline $\mathrm{H} 27 \mathrm{~B}$ & 0.543625 & -0.065338 & 0.280106 & $0.042 *$ \\
\hline $\mathrm{H} 27 \mathrm{C}$ & 0.582617 & -0.021393 & 0.319323 & $0.042 *$ \\
\hline C28 & 0.35849 (16) & $0.3024(4)$ & $0.58016(13)$ & $0.0249(9)$ \\
\hline $\mathrm{C} 29$ & $0.42995(15)$ & 0.1637 (4) & 0.43909 (11) & $0.0199(8)$ \\
\hline $\mathrm{C} 2$ & 0.37357 (17) & 0.8902 (4) & $0.53291(11)$ & $0.0284(9)$ \\
\hline $\mathrm{H} 2 \mathrm{~A}$ & 0.410648 & 0.939228 & 0.535081 & $0.043^{*}$ \\
\hline $\mathrm{H} 2 \mathrm{~B}$ & 0.342892 & 0.958133 & 0.540118 & $0.043^{*}$ \\
\hline $\mathrm{H} 2 \mathrm{C}$ & 0.368063 & 0.855479 & 0.504172 & $0.043^{*}$ \\
\hline
\end{tabular}

Atomic displacement parameters $\left(\AA^{2}\right)$

\begin{tabular}{lllllll}
\hline & $U^{11}$ & $U^{22}$ & $U^{33}$ & $U^{12}$ & $U^{13}$ & $U^{23}$ \\
\hline Fe1 & $0.0201(3)$ & $0.0141(2)$ & $0.0154(3)$ & $0.0026(2)$ & $-0.0036(2)$ & $-0.0026(2)$ \\
S1 & $0.0471(7)$ & $0.0629(8)$ & $0.0239(6)$ & $-0.0011(6)$ & $0.0011(5)$ & $0.0165(6)$ \\
S2 & $0.0348(6)$ & $0.0177(5)$ & $0.0348(6)$ & $0.0031(4)$ & $0.0071(5)$ & $-0.0057(5)$ \\
O1 & $0.0315(16)$ & $0.0343(15)$ & $0.0215(14)$ & $-0.0001(13)$ & $-0.0023(12)$ & $0.0044(13)$
\end{tabular}




\begin{tabular}{|c|c|c|c|c|c|c|}
\hline $\mathrm{O} 2$ & $0.0298(15)$ & $0.0227(13)$ & $0.0220(14)$ & $0.0095(12)$ & $0.0042(12)$ & $-0.0018(12)$ \\
\hline N1 & $0.0216(17)$ & 0.0167 (14) & $0.0151(16)$ & $0.0030(13)$ & $-0.0001(13)$ & $-0.0014(13)$ \\
\hline N2 & $0.0231(17)$ & $0.0132(14)$ & $0.0146(15)$ & $-0.0012(13)$ & $0.0007(13)$ & $0.0011(13)$ \\
\hline N3 & $0.0267(18)$ & $0.0179(15)$ & 0.0194 (17) & $-0.0017(14)$ & $-0.0055(14)$ & $-0.0015(14)$ \\
\hline N4 & $0.0231(17)$ & $0.0176(15)$ & $0.0210(17)$ & $-0.0022(13)$ & $-0.0118(14)$ & $0.0016(14)$ \\
\hline N5 & $0.0182(16)$ & $0.0145(14)$ & $0.0165(15)$ & $0.0056(12)$ & $-0.0027(13)$ & $-0.0039(14)$ \\
\hline N6 & $0.0243(17)$ & $0.0171(15)$ & $0.0148(16)$ & $0.0042(13)$ & $-0.0010(13)$ & $-0.0042(14)$ \\
\hline N7 & $0.0283(18)$ & $0.0210(16)$ & $0.0164(16)$ & $0.0027(14)$ & $-0.0008(14)$ & $-0.0031(15)$ \\
\hline N8 & $0.0300(19)$ & $0.0151(15)$ & $0.0182(17)$ & 0.0069 (14) & $0.0029(15)$ & $-0.0025(14)$ \\
\hline N9 & $0.034(2)$ & $0.0213(17)$ & $0.0221(18)$ & $-0.0045(15)$ & $-0.0018(16)$ & $0.0002(15)$ \\
\hline N10 & $0.0229(17)$ & $0.0190(16)$ & $0.0263(18)$ & 0.0049 (14) & $-0.0070(15)$ & $-0.0054(15)$ \\
\hline $\mathrm{C} 1$ & $0.038(2)$ & $0.033(2)$ & $0.022(2)$ & $0.0049(19)$ & $-0.0067(19)$ & $-0.0095(19)$ \\
\hline $\mathrm{C} 3$ & $0.025(2)$ & $0.0200(18)$ & $0.0134(18)$ & $0.0065(17)$ & $-0.0021(16)$ & $-0.0069(16)$ \\
\hline $\mathrm{C} 4$ & $0.027(2)$ & $0.028(2)$ & $0.0133(19)$ & $0.0065(17)$ & $0.0028(16)$ & -0.0028 \\
\hline $\mathrm{C} 16$ & $0.027(2)$ & $0.0182(18)$ & 0.0134 (19) & $-0.0020(16)$ & $-0.0035(16)$ & $-0.0020(16)$ \\
\hline $\mathrm{C} 5$ & $0.019(2)$ & $0.0163(17)$ & $0.021(2)$ & $0.0045(15)$ & $0.0046(16)$ & $0.0024(16)$ \\
\hline C6 & $0.0174(19)$ & $0.0148(17)$ & 0.0178 (19) & $-0.0014(15)$ & 0.0017 (16) & $0.0041(16)$ \\
\hline C7 & $0.020(2)$ & $0.0203(19)$ & $0.029(2)$ & $0.0009(16)$ & $-0.0046(18)$ & $0.0029(18)$ \\
\hline $\mathrm{C} 8$ & 0.045 & $0.023(2)$ & $0.028(2)$ & $-0.0019(19)$ & $-0.022(2)$ & $-0.0017(19)$ \\
\hline C9 & $0.036(2)$ & $0.0183(19)$ & $0.022(2)$ & $-0.0021(18)$ & $-0.0166(18)$ & $-0.0089(17)$ \\
\hline $\mathrm{C} 10$ & $0.024(2)$ & $0.026(2)$ & $0.027(2)$ & $-0.0047(18)$ & $-0.0077(17)$ & $-0.0019(19)$ \\
\hline C11 & $0.022(2)$ & $0.023(2)$ & 0.028 (2) & $0.0021(17)$ & $-0.0092(18)$ & $-0.0017(18)$ \\
\hline $\mathrm{C} 12$ & $0.029(2)$ & 0.0229 (19) & 0.0159 (19) & $0.0002(17)$ & $-0.0064(18)$ & $-0.0053(17)$ \\
\hline $\mathrm{C} 13$ & $0.027(2)$ & $0.033(2)$ & $0.024(2)$ & $0.0076(18)$ & $0.0018(18)$ & $-0.0093(19)$ \\
\hline $\mathrm{C} 14$ & $0.030(2)$ & $0.024(2)$ & $0.031(2)$ & $0.0126(18)$ & $-0.012(2)$ & $-0.0089(19)$ \\
\hline $\mathrm{C} 15$ & $0.034(3)$ & $0.057(3)$ & $0.029(2)$ & $-0.003(2)$ & 0.007 (2) & $0.005(2)$ \\
\hline $\mathrm{C} 17$ & $0.0162(19)$ & 0.0157 (17) & $0.024(2)$ & $0.0031(15)$ & $-0.0044(17)$ & $0.0006(17)$ \\
\hline $\mathrm{C} 18$ & $0.0151(19)$ & $0.0110(16)$ & $0.022(2)$ & $0.0042(15)$ & $-0.0013(16)$ & $-0.0035(16)$ \\
\hline $\mathrm{C} 19$ & $0.023(2)$ & $0.0131(17)$ & $0.025(2)$ & $0.0050(16)$ & $0.0023(17)$ & $0.0012(17)$ \\
\hline $\mathrm{C} 20$ & 0.043 & $0.030(2)$ & $0.014(2)$ & $0.0088(19)$ & 0.0047 (18) & $0.0024(18)$ \\
\hline $\mathrm{C} 21$ & $0.029(2)$ & $0.0201(19)$ & 0.0099 (18) & $0.0015(17)$ & $-0.0038(16)$ & $0.0009(16)$ \\
\hline $\mathrm{C} 22$ & $0.033(2)$ & $0.0198(19)$ & 0.0166 (19) & $-0.0028(17)$ & $0.0020(18)$ & $0.0039(17)$ \\
\hline $\mathrm{C} 23$ & $0.023(2)$ & $0.028(2)$ & $0.018(2)$ & $0.0018(17)$ & 0.0064 (17) & $-0.0004(18)$ \\
\hline $\mathrm{C} 24$ & $0.024(2)$ & $0.0229(19)$ & $0.0105(18)$ & $0.0024(17)$ & $-0.0032(16)$ & $-0.0051(16)$ \\
\hline $\mathrm{C} 25$ & $0.029(2)$ & $0.0167(18)$ & $0.020(2)$ & $0.0011(17)$ & 0.0007 (17) & $-0.0003(17)$ \\
\hline $\mathrm{C} 26$ & $0.026(2)$ & $0.028(2)$ & $0.015(2)$ & $-0.0004(18)$ & $0.0024(16)$ & $0.0021(18)$ \\
\hline $\mathrm{C} 27$ & $0.042(2)$ & $0.021(2)$ & $0.022(2)$ & $0.0107(18)$ & $0.0012(19)$ & $0.0001(17)$ \\
\hline $\mathrm{C} 28$ & $0.023(2)$ & $0.0181(19)$ & $0.034(2)$ & $-0.0012(16)$ & $-0.0035(19)$ & $0.0049(19)$ \\
\hline $\mathrm{C} 29$ & $0.019(2)$ & 0.0207 (19) & $0.020(2)$ & $-0.0035(16)$ & $-0.0035(16)$ & $0.0056(18)$ \\
\hline $\mathrm{C} 2$ & $0.040(2)$ & $0.0204(19)$ & $0.025(2)$ & $0.0086(18)$ & $-0.0059(19)$ & $-0.0034(18)$ \\
\hline
\end{tabular}

Geometric parameters $\left(\AA,{ }^{o}\right)$

\begin{tabular}{llll}
\hline $\mathrm{Fe} 1-\mathrm{N} 1$ & $2.242(3)$ & $\mathrm{C} 6-\mathrm{C} 7$ & $1.365(5)$ \\
$\mathrm{Fe} 1-\mathrm{N} 2$ & $2.138(3)$ & $\mathrm{C} 7-\mathrm{H} 7$ & 0.9300 \\
$\mathrm{Fe} 1-\mathrm{N} 5$ & $2.167(3)$ & $\mathrm{C} 8-\mathrm{H} 8 \mathrm{~A}$ & 0.9700 \\
$\mathrm{Fe} 1-\mathrm{N} 6$ & $2.288(3)$ & $\mathrm{C} 8-\mathrm{H} 8 \mathrm{~B}$ & 0.9700 \\
$\mathrm{Fe} 1-\mathrm{N} 9$ & $2.073(3)$ & $\mathrm{C} 8-\mathrm{C} 9$ & $1.512(5)$
\end{tabular}




\begin{tabular}{|c|c|c|c|}
\hline $\mathrm{Fe} 1-\mathrm{N} 10$ & $2.092(3)$ & $\mathrm{C} 9-\mathrm{C} 10$ & $1.389(5)$ \\
\hline $\mathrm{S} 1-\mathrm{C} 28$ & $1.634(4)$ & $\mathrm{C} 9-\mathrm{C} 14$ & $1.384(5)$ \\
\hline $\mathrm{S} 2-\mathrm{C} 29$ & $1.633(4)$ & $\mathrm{C} 10-\mathrm{H} 10$ & 0.9300 \\
\hline $\mathrm{O} 1-\mathrm{C} 12$ & $1.371(4)$ & $\mathrm{C} 10-\mathrm{C} 11$ & $1.369(5)$ \\
\hline $\mathrm{O} 1-\mathrm{C} 15$ & $1.434(4)$ & $\mathrm{C} 11-\mathrm{H} 11$ & 0.9300 \\
\hline $\mathrm{O} 2-\mathrm{C} 24$ & $1.373(4)$ & $\mathrm{C} 11-\mathrm{C} 12$ & $1.385(5)$ \\
\hline $\mathrm{O} 2-\mathrm{C} 27$ & $1.435(4)$ & $\mathrm{C} 12-\mathrm{C} 13$ & $1.382(5)$ \\
\hline $\mathrm{N} 1-\mathrm{C} 4$ & $1.475(4)$ & $\mathrm{C} 13-\mathrm{H} 13$ & 0.9300 \\
\hline $\mathrm{N} 1-\mathrm{C} 5$ & $1.272(4)$ & $\mathrm{C} 13-\mathrm{C} 14$ & $1.397(5)$ \\
\hline $\mathrm{N} 2-\mathrm{N} 3$ & $1.319(4)$ & $\mathrm{C} 14-\mathrm{H} 14$ & 0.9300 \\
\hline $\mathrm{N} 2-\mathrm{C} 6$ & $1.362(4)$ & $\mathrm{C} 15-\mathrm{H} 15 \mathrm{~A}$ & 0.9600 \\
\hline $\mathrm{N} 3-\mathrm{N} 4$ & $1.344(4)$ & C15-H15B & 0.9600 \\
\hline $\mathrm{N} 4-\mathrm{C} 7$ & $1.345(4)$ & $\mathrm{C} 15-\mathrm{H} 15 \mathrm{C}$ & 0.9600 \\
\hline $\mathrm{N} 4-\mathrm{C} 8$ & $1.470(4)$ & C17-H17 & 0.9300 \\
\hline N5-C16 & $1.469(4)$ & $\mathrm{C} 17-\mathrm{C} 18$ & $1.447(4)$ \\
\hline $\mathrm{N} 5-\mathrm{C} 17$ & $1.279(4)$ & $\mathrm{C} 18-\mathrm{C} 19$ & $1.366(4)$ \\
\hline $\mathrm{N} 6-\mathrm{N} 7$ & $1.311(4)$ & C19-H19 & 0.9300 \\
\hline N6-C18 & $1.355(4)$ & $\mathrm{C} 20-\mathrm{H} 20 \mathrm{~A}$ & 0.9700 \\
\hline $\mathrm{N} 7-\mathrm{N} 8$ & $1.350(4)$ & $\mathrm{C} 20-\mathrm{H} 20 \mathrm{~B}$ & 0.9700 \\
\hline $\mathrm{N} 8-\mathrm{C} 19$ & $1.346(4)$ & $\mathrm{C} 20-\mathrm{C} 21$ & $1.508(5)$ \\
\hline $\mathrm{N} 8-\mathrm{C} 20$ & $1.474(4)$ & $\mathrm{C} 21-\mathrm{C} 22$ & $1.391(5)$ \\
\hline N9- $\mathrm{C} 28$ & $1.157(4)$ & $\mathrm{C} 21-\mathrm{C} 26$ & $1.384(5)$ \\
\hline $\mathrm{N} 10-\mathrm{C} 29$ & $1.164(4)$ & $\mathrm{C} 22-\mathrm{H} 22$ & 0.9300 \\
\hline $\mathrm{C} 1-\mathrm{H} 1 \mathrm{~A}$ & 0.9600 & $\mathrm{C} 22-\mathrm{C} 23$ & $1.373(5)$ \\
\hline $\mathrm{C} 1-\mathrm{H} 1 \mathrm{~B}$ & 0.9600 & $\mathrm{C} 23-\mathrm{H} 23$ & 0.9300 \\
\hline $\mathrm{C} 1-\mathrm{H} 1 \mathrm{C}$ & 0.9600 & $\mathrm{C} 23-\mathrm{C} 24$ & $1.388(5)$ \\
\hline $\mathrm{C} 1-\mathrm{C} 3$ & $1.524(4)$ & $\mathrm{C} 24-\mathrm{C} 25$ & $1.379(5)$ \\
\hline $\mathrm{C} 3-\mathrm{C} 4$ & $1.531(5)$ & $\mathrm{C} 25-\mathrm{H} 25$ & 0.9300 \\
\hline $\mathrm{C} 3-\mathrm{C} 16$ & $1.549(4)$ & $\mathrm{C} 25-\mathrm{C} 26$ & $1.389(5)$ \\
\hline $\mathrm{C} 3-\mathrm{C} 2$ & $1.532(5)$ & $\mathrm{C} 26-\mathrm{H} 26$ & 0.9300 \\
\hline $\mathrm{C} 4-\mathrm{H} 4 \mathrm{~A}$ & 0.9700 & $\mathrm{C} 27-\mathrm{H} 27 \mathrm{~A}$ & 0.9600 \\
\hline $\mathrm{C} 4-\mathrm{H} 4 \mathrm{~B}$ & 0.9700 & $\mathrm{C} 27-\mathrm{H} 27 \mathrm{~B}$ & 0.9600 \\
\hline C16-H16A & 0.9700 & $\mathrm{C} 27-\mathrm{H} 27 \mathrm{C}$ & 0.9600 \\
\hline C16-H16B & 0.9700 & $\mathrm{C} 2-\mathrm{H} 2 \mathrm{~A}$ & 0.9600 \\
\hline $\mathrm{C} 5-\mathrm{H} 5$ & 0.9300 & $\mathrm{C} 2-\mathrm{H} 2 \mathrm{~B}$ & 0.9600 \\
\hline $\mathrm{C} 5-\mathrm{C} 6$ & $1.447(5)$ & $\mathrm{C} 2-\mathrm{H} 2 \mathrm{C}$ & 0.9600 \\
\hline $\mathrm{N} 1-\mathrm{Fe} 1-\mathrm{N} 6$ & $103.14(10)$ & $\mathrm{H} 8 \mathrm{~A}-\mathrm{C} 8-\mathrm{H} 8 \mathrm{~B}$ & 108.0 \\
\hline $\mathrm{N} 2-\mathrm{Fe} 1-\mathrm{N} 1$ & $74.82(10)$ & $\mathrm{C} 9-\mathrm{C} 8-\mathrm{H} 8 \mathrm{~A}$ & 109.4 \\
\hline $\mathrm{N} 2-\mathrm{Fe} 1-\mathrm{N} 5$ & $141.53(10)$ & $\mathrm{C} 9-\mathrm{C} 8-\mathrm{H} 8 \mathrm{~B}$ & 109.4 \\
\hline $\mathrm{N} 2-\mathrm{Fe} 1-\mathrm{N} 6$ & $84.71(10)$ & $\mathrm{C} 10-\mathrm{C} 9-\mathrm{C} 8$ & $121.2(4)$ \\
\hline $\mathrm{N} 5-\mathrm{Fe} 1-\mathrm{N} 1$ & $80.01(10)$ & $\mathrm{C} 14-\mathrm{C} 9-\mathrm{C} 8$ & $121.1(3)$ \\
\hline $\mathrm{N} 5-\mathrm{Fe} 1-\mathrm{N} 6$ & $73.16(10)$ & $\mathrm{C} 14-\mathrm{C} 9-\mathrm{C} 10$ & $117.8(3)$ \\
\hline $\mathrm{N} 9-\mathrm{Fe} 1-\mathrm{N} 1$ & $85.69(11)$ & $\mathrm{C} 9-\mathrm{C} 10-\mathrm{H} 10$ & 119.3 \\
\hline $\mathrm{N} 9-\mathrm{Fe} 1-\mathrm{N} 2$ & $110.72(11)$ & $\mathrm{C} 11-\mathrm{C} 10-\mathrm{C} 9$ & $121.4(4)$ \\
\hline N9-Fe1-N5 & $95.66(11)$ & $\mathrm{C} 11-\mathrm{C} 10-\mathrm{H} 10$ & 119.3 \\
\hline $\mathrm{N} 9-\mathrm{Fe} 1-\mathrm{N} 6$ & $163.98(11)$ & $\mathrm{C} 10-\mathrm{C} 11-\mathrm{H} 11$ & 119.8 \\
\hline $\mathrm{N} 9-\mathrm{Fe} 1-\mathrm{N} 10$ & $91.62(11)$ & $\mathrm{C} 10-\mathrm{C} 11-\mathrm{C} 12$ & $120.3(3)$ \\
\hline
\end{tabular}




\begin{tabular}{|c|c|c|c|}
\hline $\mathrm{N} 10-\mathrm{Fe} 1-\mathrm{N} 1$ & $167.02(11)$ & $\mathrm{C} 12-\mathrm{C} 11-\mathrm{H} 11$ & 119.8 \\
\hline $\mathrm{N} 10-\mathrm{Fe} 1-\mathrm{N} 2$ & $94.38(11)$ & $\mathrm{O} 1-\mathrm{C} 12-\mathrm{C} 11$ & $115.8(3)$ \\
\hline $\mathrm{N} 10-\mathrm{Fe} 1-\mathrm{N} 5$ & $112.91(10)$ & $\mathrm{O} 1-\mathrm{C} 12-\mathrm{C} 13$ & $124.5(3)$ \\
\hline $\mathrm{N} 10-\mathrm{Fe} 1-\mathrm{N} 6$ & $82.61(10)$ & $\mathrm{C} 13-\mathrm{C} 12-\mathrm{C} 11$ & $119.7(3)$ \\
\hline $\mathrm{C} 12-\mathrm{O} 1-\mathrm{C} 15$ & $117.6(3)$ & $\mathrm{C} 12-\mathrm{C} 13-\mathrm{H} 13$ & 120.4 \\
\hline $\mathrm{C} 24-\mathrm{O} 2-\mathrm{C} 27$ & $117.5(3)$ & $\mathrm{C} 12-\mathrm{C} 13-\mathrm{C} 14$ & $119.2(4)$ \\
\hline $\mathrm{C} 4-\mathrm{N} 1-\mathrm{Fe} 1$ & $124.6(2)$ & $\mathrm{C} 14-\mathrm{C} 13-\mathrm{H} 13$ & 120.4 \\
\hline $\mathrm{C} 5-\mathrm{N} 1-\mathrm{Fe} 1$ & $115.3(2)$ & $\mathrm{C} 9-\mathrm{C} 14-\mathrm{C} 13$ & $121.5(3)$ \\
\hline $\mathrm{C} 5-\mathrm{N} 1-\mathrm{C} 4$ & $119.4(3)$ & $\mathrm{C} 9-\mathrm{C} 14-\mathrm{H} 14$ & 119.3 \\
\hline $\mathrm{N} 3-\mathrm{N} 2-\mathrm{Fe} 1$ & $134.6(2)$ & $\mathrm{C} 13-\mathrm{C} 14-\mathrm{H} 14$ & 119.3 \\
\hline $\mathrm{N} 3-\mathrm{N} 2-\mathrm{C} 6$ & $110.1(3)$ & $\mathrm{O} 1-\mathrm{C} 15-\mathrm{H} 15 \mathrm{~A}$ & 109.5 \\
\hline $\mathrm{C} 6-\mathrm{N} 2-\mathrm{Fe} 1$ & $114.6(2)$ & $\mathrm{O} 1-\mathrm{C} 15-\mathrm{H} 15 \mathrm{~B}$ & 109.5 \\
\hline $\mathrm{N} 2-\mathrm{N} 3-\mathrm{N} 4$ & $105.6(3)$ & $\mathrm{O} 1-\mathrm{C} 15-\mathrm{H} 15 \mathrm{C}$ & 109.5 \\
\hline $\mathrm{N} 3-\mathrm{N} 4-\mathrm{C} 7$ & $111.8(3)$ & $\mathrm{H} 15 \mathrm{~A}-\mathrm{C} 15-\mathrm{H} 15 \mathrm{~B}$ & 109.5 \\
\hline $\mathrm{N} 3-\mathrm{N} 4-\mathrm{C} 8$ & $119.6(3)$ & $\mathrm{H} 15 \mathrm{~A}-\mathrm{C} 15-\mathrm{H} 15 \mathrm{C}$ & 109.5 \\
\hline $\mathrm{C} 7-\mathrm{N} 4-\mathrm{C} 8$ & $128.5(3)$ & $\mathrm{H} 15 \mathrm{~B}-\mathrm{C} 15-\mathrm{H} 15 \mathrm{C}$ & 109.5 \\
\hline $\mathrm{C} 16-\mathrm{N} 5-\mathrm{Fe} 1$ & $122.3(2)$ & $\mathrm{N} 5-\mathrm{C} 17-\mathrm{H} 17$ & 121.3 \\
\hline $\mathrm{C} 17-\mathrm{N} 5-\mathrm{Fe} 1$ & $117.8(2)$ & N5-C17-C18 & $117.5(3)$ \\
\hline $\mathrm{C} 17-\mathrm{N} 5-\mathrm{C} 16$ & $118.9(3)$ & $\mathrm{C} 18-\mathrm{C} 17-\mathrm{H} 17$ & 121.3 \\
\hline $\mathrm{N} 7-\mathrm{N} 6-\mathrm{Fe} 1$ & $135.2(2)$ & $\mathrm{N} 6-\mathrm{C} 18-\mathrm{C} 17$ & $116.0(3)$ \\
\hline $\mathrm{N} 7-\mathrm{N} 6-\mathrm{C} 18$ & $109.8(3)$ & $\mathrm{N} 6-\mathrm{C} 18-\mathrm{C} 19$ & $108.3(3)$ \\
\hline $\mathrm{C} 18-\mathrm{N} 6-\mathrm{Fe} 1$ & $109.6(2)$ & $\mathrm{C} 19-\mathrm{C} 18-\mathrm{C} 17$ & $135.5(3)$ \\
\hline $\mathrm{N} 6-\mathrm{N} 7-\mathrm{N} 8$ & $106.0(3)$ & $\mathrm{N} 8-\mathrm{C} 19-\mathrm{C} 18$ & $104.4(3)$ \\
\hline $\mathrm{N} 7-\mathrm{N} 8-\mathrm{C} 20$ & $119.2(3)$ & N8-C19-H19 & 127.8 \\
\hline $\mathrm{C} 19-\mathrm{N} 8-\mathrm{N} 7$ & $111.5(3)$ & $\mathrm{C} 18-\mathrm{C} 19-\mathrm{H} 19$ & 127.8 \\
\hline $\mathrm{C} 19-\mathrm{N} 8-\mathrm{C} 20$ & $129.2(3)$ & $\mathrm{N} 8-\mathrm{C} 20-\mathrm{H} 20 \mathrm{~A}$ & 109.0 \\
\hline $\mathrm{C} 28-\mathrm{N} 9-\mathrm{Fe} 1$ & $157.3(3)$ & $\mathrm{N} 8-\mathrm{C} 20-\mathrm{H} 20 \mathrm{~B}$ & 109.0 \\
\hline $\mathrm{C} 29-\mathrm{N} 10-\mathrm{Fe} 1$ & $174.6(3)$ & $\mathrm{N} 8-\mathrm{C} 20-\mathrm{C} 21$ & $112.9(3)$ \\
\hline $\mathrm{H} 1 \mathrm{~A}-\mathrm{C} 1-\mathrm{H} 1 \mathrm{~B}$ & 109.5 & $\mathrm{H} 20 \mathrm{~A}-\mathrm{C} 20-\mathrm{H} 20 \mathrm{~B}$ & 107.8 \\
\hline $\mathrm{H} 1 \mathrm{~A}-\mathrm{C} 1-\mathrm{H} 1 \mathrm{C}$ & 109.5 & $\mathrm{C} 21-\mathrm{C} 20-\mathrm{H} 20 \mathrm{~A}$ & 109.0 \\
\hline $\mathrm{H} 1 \mathrm{~B}-\mathrm{C} 1-\mathrm{H} 1 \mathrm{C}$ & 109.5 & $\mathrm{C} 21-\mathrm{C} 20-\mathrm{H} 20 \mathrm{~B}$ & 109.0 \\
\hline $\mathrm{C} 3-\mathrm{C} 1-\mathrm{H} 1 \mathrm{~A}$ & 109.5 & $\mathrm{C} 22-\mathrm{C} 21-\mathrm{C} 20$ & $119.9(3)$ \\
\hline $\mathrm{C} 3-\mathrm{C} 1-\mathrm{H} 1 \mathrm{~B}$ & 109.5 & $\mathrm{C} 26-\mathrm{C} 21-\mathrm{C} 20$ & $121.7(3)$ \\
\hline $\mathrm{C} 3-\mathrm{C} 1-\mathrm{H} 1 \mathrm{C}$ & 109.5 & $\mathrm{C} 26-\mathrm{C} 21-\mathrm{C} 22$ & $118.4(3)$ \\
\hline $\mathrm{C} 1-\mathrm{C} 3-\mathrm{C} 4$ & $107.3(3)$ & $\mathrm{C} 21-\mathrm{C} 22-\mathrm{H} 22$ & 119.7 \\
\hline $\mathrm{C} 1-\mathrm{C} 3-\mathrm{C} 16$ & $106.6(3)$ & $\mathrm{C} 23-\mathrm{C} 22-\mathrm{C} 21$ & $120.7(3)$ \\
\hline $\mathrm{C} 1-\mathrm{C} 3-\mathrm{C} 2$ & $109.2(3)$ & $\mathrm{C} 23-\mathrm{C} 22-\mathrm{H} 22$ & 119.7 \\
\hline $\mathrm{C} 4-\mathrm{C} 3-\mathrm{C} 16$ & $112.0(3)$ & $\mathrm{C} 22-\mathrm{C} 23-\mathrm{H} 23$ & 119.7 \\
\hline $\mathrm{C} 4-\mathrm{C} 3-\mathrm{C} 2$ & $110.8(3)$ & $\mathrm{C} 22-\mathrm{C} 23-\mathrm{C} 24$ & $120.7(3)$ \\
\hline $\mathrm{C} 2-\mathrm{C} 3-\mathrm{C} 16$ & $110.8(3)$ & $\mathrm{C} 24-\mathrm{C} 23-\mathrm{H} 23$ & 119.7 \\
\hline $\mathrm{N} 1-\mathrm{C} 4-\mathrm{C} 3$ & $114.6(3)$ & $\mathrm{O} 2-\mathrm{C} 24-\mathrm{C} 23$ & $115.8(3)$ \\
\hline $\mathrm{N} 1-\mathrm{C} 4-\mathrm{H} 4 \mathrm{~A}$ & 108.6 & $\mathrm{O} 2-\mathrm{C} 24-\mathrm{C} 25$ & $124.9(3)$ \\
\hline $\mathrm{N} 1-\mathrm{C} 4-\mathrm{H} 4 \mathrm{~B}$ & 108.6 & $\mathrm{C} 25-\mathrm{C} 24-\mathrm{C} 23$ & $119.3(3)$ \\
\hline $\mathrm{C} 3-\mathrm{C} 4-\mathrm{H} 4 \mathrm{~A}$ & 108.6 & $\mathrm{C} 24-\mathrm{C} 25-\mathrm{H} 25$ & 120.1 \\
\hline $\mathrm{C} 3-\mathrm{C} 4-\mathrm{H} 4 \mathrm{~B}$ & 108.6 & $\mathrm{C} 24-\mathrm{C} 25-\mathrm{C} 26$ & $119.8(3)$ \\
\hline $\mathrm{H} 4 \mathrm{~A}-\mathrm{C} 4-\mathrm{H} 4 \mathrm{~B}$ & 107.6 & $\mathrm{C} 26-\mathrm{C} 25-\mathrm{H} 25$ & 120.1 \\
\hline $\mathrm{N} 5-\mathrm{C} 16-\mathrm{C} 3$ & $111.7(3)$ & $\mathrm{C} 21-\mathrm{C} 26-\mathrm{C} 25$ & $121.2(3)$ \\
\hline $\mathrm{N} 5-\mathrm{C} 16-\mathrm{H} 16 \mathrm{~A}$ & 109.3 & $\mathrm{C} 21-\mathrm{C} 26-\mathrm{H} 26$ & 119.4 \\
\hline
\end{tabular}




\begin{tabular}{|c|c|c|c|}
\hline $\mathrm{N} 5-\mathrm{C} 16-\mathrm{H} 16 \mathrm{~B}$ & 109.3 & $\mathrm{C} 25-\mathrm{C} 26-\mathrm{H} 26$ & 119.4 \\
\hline $\mathrm{C} 3-\mathrm{C} 16-\mathrm{H} 16 \mathrm{~A}$ & 109.3 & $\mathrm{O} 2-\mathrm{C} 27-\mathrm{H} 27 \mathrm{~A}$ & 109.5 \\
\hline $\mathrm{C} 3-\mathrm{C} 16-\mathrm{H} 16 \mathrm{~B}$ & 109.3 & $\mathrm{O} 2-\mathrm{C} 27-\mathrm{H} 27 \mathrm{~B}$ & 109.5 \\
\hline $\mathrm{H} 16 \mathrm{~A}-\mathrm{C} 16-\mathrm{H} 16 \mathrm{~B}$ & 107.9 & $\mathrm{O} 2-\mathrm{C} 27-\mathrm{H} 27 \mathrm{C}$ & 109.5 \\
\hline $\mathrm{N} 1-\mathrm{C} 5-\mathrm{H} 5$ & 121.6 & $\mathrm{H} 27 \mathrm{~A}-\mathrm{C} 27-\mathrm{H} 27 \mathrm{~B}$ & 109.5 \\
\hline $\mathrm{N} 1-\mathrm{C} 5-\mathrm{C} 6$ & $116.8(3)$ & $\mathrm{H} 27 \mathrm{~A}-\mathrm{C} 27-\mathrm{H} 27 \mathrm{C}$ & 109.5 \\
\hline $\mathrm{C} 6-\mathrm{C} 5-\mathrm{H} 5$ & 121.6 & $\mathrm{H} 27 \mathrm{~B}-\mathrm{C} 27-\mathrm{H} 27 \mathrm{C}$ & 109.5 \\
\hline $\mathrm{N} 2-\mathrm{C} 6-\mathrm{C} 5$ & $118.0(3)$ & $\mathrm{N} 9-\mathrm{C} 28-\mathrm{S} 1$ & $178.7(4)$ \\
\hline $\mathrm{N} 2-\mathrm{C} 6-\mathrm{C} 7$ & $107.5(3)$ & $\mathrm{N} 10-\mathrm{C} 29-\mathrm{S} 2$ & $178.7(3)$ \\
\hline $\mathrm{C} 7-\mathrm{C} 6-\mathrm{C} 5$ & $134.6(3)$ & $\mathrm{C} 3-\mathrm{C} 2-\mathrm{H} 2 \mathrm{~A}$ & 109.5 \\
\hline $\mathrm{N} 4-\mathrm{C} 7-\mathrm{C} 6$ & $105.0(3)$ & $\mathrm{C} 3-\mathrm{C} 2-\mathrm{H} 2 \mathrm{~B}$ & 109.5 \\
\hline $\mathrm{N} 4-\mathrm{C} 7-\mathrm{H} 7$ & 127.5 & $\mathrm{C} 3-\mathrm{C} 2-\mathrm{H} 2 \mathrm{C}$ & 109.5 \\
\hline $\mathrm{C} 6-\mathrm{C} 7-\mathrm{H} 7$ & 127.5 & $\mathrm{H} 2 \mathrm{~A}-\mathrm{C} 2-\mathrm{H} 2 \mathrm{~B}$ & 109.5 \\
\hline $\mathrm{N} 4-\mathrm{C} 8-\mathrm{H} 8 \mathrm{~A}$ & 109.4 & $\mathrm{H} 2 \mathrm{~A}-\mathrm{C} 2-\mathrm{H} 2 \mathrm{C}$ & 109.5 \\
\hline $\mathrm{N} 4-\mathrm{C} 8-\mathrm{H} 8 \mathrm{~B}$ & 109.4 & $\mathrm{H} 2 \mathrm{~B}-\mathrm{C} 2-\mathrm{H} 2 \mathrm{C}$ & 109.5 \\
\hline $\mathrm{N} 4-\mathrm{C} 8-\mathrm{C} 9$ & $111.3(3)$ & & \\
\hline $\mathrm{Fe} 1-\mathrm{N} 1-\mathrm{C} 4-\mathrm{C} 3$ & $55.4(4)$ & $\mathrm{C} 4-\mathrm{C} 3-\mathrm{C} 16-\mathrm{N} 5$ & $66.6(4)$ \\
\hline $\mathrm{Fe} 1-\mathrm{N} 1-\mathrm{C} 5-\mathrm{C} 6$ & $-1.1(4)$ & $\mathrm{C} 16-\mathrm{N} 5-\mathrm{C} 17-\mathrm{C} 18$ & $-165.6(3)$ \\
\hline $\mathrm{Fe} 1-\mathrm{N} 2-\mathrm{N} 3-\mathrm{N} 4$ & $-170.5(2)$ & $\mathrm{C} 16-\mathrm{C} 3-\mathrm{C} 4-\mathrm{N} 1$ & $-59.6(4)$ \\
\hline $\mathrm{Fe} 1-\mathrm{N} 2-\mathrm{C} 6-\mathrm{C} 5$ & $-8.2(4)$ & $\mathrm{C} 5-\mathrm{N} 1-\mathrm{C} 4-\mathrm{C} 3$ & $-134.5(3)$ \\
\hline $\mathrm{Fe} 1-\mathrm{N} 2-\mathrm{C} 6-\mathrm{C} 7$ & $172.3(2)$ & $\mathrm{C} 5-\mathrm{C} 6-\mathrm{C} 7-\mathrm{N} 4$ & $-179.2(4)$ \\
\hline $\mathrm{Fe} 1-\mathrm{N} 5-\mathrm{C} 16-\mathrm{C} 3$ & $-70.4(3)$ & $\mathrm{C} 6-\mathrm{N} 2-\mathrm{N} 3-\mathrm{N} 4$ & $-0.9(3)$ \\
\hline $\mathrm{Fe} 1-\mathrm{N} 5-\mathrm{C} 17-\mathrm{C} 18$ & $3.1(4)$ & $\mathrm{C} 7-\mathrm{N} 4-\mathrm{C} 8-\mathrm{C} 9$ & $73.0(5)$ \\
\hline $\mathrm{Fe} 1-\mathrm{N} 6-\mathrm{N} 7-\mathrm{N} 8$ & $-150.2(2)$ & $\mathrm{C} 8-\mathrm{N} 4-\mathrm{C} 7-\mathrm{C} 6$ & $-176.5(3)$ \\
\hline $\mathrm{Fe} 1-\mathrm{N} 6-\mathrm{C} 18-\mathrm{C} 17$ & $-26.4(3)$ & $\mathrm{C} 8-\mathrm{C} 9-\mathrm{C} 10-\mathrm{C} 11$ & $-178.1(3)$ \\
\hline $\mathrm{Fe} 1-\mathrm{N} 6-\mathrm{C} 18-\mathrm{C} 19$ & $157.9(2)$ & $\mathrm{C} 8-\mathrm{C} 9-\mathrm{C} 14-\mathrm{C} 13$ & $178.2(3)$ \\
\hline $\mathrm{O} 1-\mathrm{C} 12-\mathrm{C} 13-\mathrm{C} 14$ & $-177.7(3)$ & $\mathrm{C} 9-\mathrm{C} 10-\mathrm{C} 11-\mathrm{C} 12$ & $0.2(5)$ \\
\hline $\mathrm{O} 2-\mathrm{C} 24-\mathrm{C} 25-\mathrm{C} 26$ & $177.8(3)$ & $\mathrm{C} 10-\mathrm{C} 9-\mathrm{C} 14-\mathrm{C} 13$ & $-2.2(5)$ \\
\hline $\mathrm{N} 1-\mathrm{C} 5-\mathrm{C} 6-\mathrm{N} 2$ & $6.2(5)$ & $\mathrm{C} 10-\mathrm{C} 11-\mathrm{C} 12-\mathrm{O} 1$ & $177.8(3)$ \\
\hline $\mathrm{N} 1-\mathrm{C} 5-\mathrm{C} 6-\mathrm{C} 7$ & $-174.5(4)$ & $\mathrm{C} 10-\mathrm{C} 11-\mathrm{C} 12-\mathrm{C} 13$ & $-2.8(5)$ \\
\hline $\mathrm{N} 2-\mathrm{N} 3-\mathrm{N} 4-\mathrm{C} 7$ & $1.0(4)$ & $\mathrm{C} 11-\mathrm{C} 12-\mathrm{C} 13-\mathrm{C} 14$ & $2.9(5)$ \\
\hline $\mathrm{N} 2-\mathrm{N} 3-\mathrm{N} 4-\mathrm{C} 8$ & $177.2(3)$ & $\mathrm{C} 12-\mathrm{C} 13-\mathrm{C} 14-\mathrm{C} 9$ & $-0.4(5)$ \\
\hline $\mathrm{N} 2-\mathrm{C} 6-\mathrm{C} 7-\mathrm{N} 4$ & $0.1(4)$ & $\mathrm{C} 14-\mathrm{C} 9-\mathrm{C} 10-\mathrm{C} 11$ & $2.3(5)$ \\
\hline $\mathrm{N} 3-\mathrm{N} 2-\mathrm{C} 6-\mathrm{C} 5$ & $179.9(3)$ & $\mathrm{C} 15-\mathrm{O} 1-\mathrm{C} 12-\mathrm{C} 11$ & $179.9(3)$ \\
\hline $\mathrm{N} 3-\mathrm{N} 2-\mathrm{C} 6-\mathrm{C} 7$ & $0.5(4)$ & $\mathrm{C} 15-\mathrm{O} 1-\mathrm{C} 12-\mathrm{C} 13$ & $0.5(5)$ \\
\hline $\mathrm{N} 3-\mathrm{N} 4-\mathrm{C} 7-\mathrm{C} 6$ & $-0.7(4)$ & $\mathrm{C} 17-\mathrm{N} 5-\mathrm{C} 16-\mathrm{C} 3$ & $97.8(3)$ \\
\hline $\mathrm{N} 3-\mathrm{N} 4-\mathrm{C} 8-\mathrm{C} 9$ & $-102.6(4)$ & $\mathrm{C} 17-\mathrm{C} 18-\mathrm{C} 19-\mathrm{N} 8$ & $-174.1(3)$ \\
\hline $\mathrm{N} 4-\mathrm{C} 8-\mathrm{C} 9-\mathrm{C} 10$ & $-104.9(4)$ & $\mathrm{C} 18-\mathrm{N} 6-\mathrm{N} 7-\mathrm{N} 8$ & $0.0(3)$ \\
\hline $\mathrm{N} 4-\mathrm{C} 8-\mathrm{C} 9-\mathrm{C} 14$ & $74.7(4)$ & $\mathrm{C} 19-\mathrm{N} 8-\mathrm{C} 20-\mathrm{C} 21$ & $86.0(4)$ \\
\hline $\mathrm{N} 5-\mathrm{C} 17-\mathrm{C} 18-\mathrm{N} 6$ & $17.1(4)$ & $\mathrm{C} 20-\mathrm{N} 8-\mathrm{C} 19-\mathrm{C} 18$ & $-177.8(3)$ \\
\hline $\mathrm{N} 5-\mathrm{C} 17-\mathrm{C} 18-\mathrm{C} 19$ & $-168.7(4)$ & $\mathrm{C} 20-\mathrm{C} 21-\mathrm{C} 22-\mathrm{C} 23$ & $-178.4(3)$ \\
\hline N6-N7-N8-C19 & $0.2(3)$ & $\mathrm{C} 20-\mathrm{C} 21-\mathrm{C} 26-\mathrm{C} 25$ & $179.2(3)$ \\
\hline $\mathrm{N} 6-\mathrm{N} 7-\mathrm{N} 8-\mathrm{C} 20$ & $177.9(3)$ & $\mathrm{C} 21-\mathrm{C} 22-\mathrm{C} 23-\mathrm{C} 24$ & $-1.0(5)$ \\
\hline $\mathrm{N} 6-\mathrm{C} 18-\mathrm{C} 19-\mathrm{N} 8$ & $0.4(4)$ & $\mathrm{C} 22-\mathrm{C} 21-\mathrm{C} 26-\mathrm{C} 25$ & $0.7(5)$ \\
\hline $\mathrm{N} 7-\mathrm{N} 6-\mathrm{C} 18-\mathrm{C} 17$ & $175.4(3)$ & $\mathrm{C} 22-\mathrm{C} 23-\mathrm{C} 24-\mathrm{O} 2$ & $-177.1(3)$ \\
\hline N7-N6-C18-C19 & $-0.3(4)$ & $\mathrm{C} 22-\mathrm{C} 23-\mathrm{C} 24-\mathrm{C} 25$ & $1.0(5)$ \\
\hline $\mathrm{N} 7-\mathrm{N} 8-\mathrm{C} 19-\mathrm{C} 18$ & $-0.4(4)$ & $\mathrm{C} 23-\mathrm{C} 24-\mathrm{C} 25-\mathrm{C} 26$ & $-0.1(5)$ \\
\hline
\end{tabular}




$\begin{array}{llll}\mathrm{N} 7-\mathrm{N} 8-\mathrm{C} 20-\mathrm{C} 21 & -91.3(4) & \mathrm{C} 24-\mathrm{C} 25-\mathrm{C} 26-\mathrm{C} 21 & -0.7(5) \\ \mathrm{N} 8-\mathrm{C} 20-\mathrm{C} 21-\mathrm{C} 22 & -133.2(3) & \mathrm{C} 26-\mathrm{C} 21-\mathrm{C} 22-\mathrm{C} 23 & 0.2(5) \\ \mathrm{N} 8-\mathrm{C} 20-\mathrm{C} 21-\mathrm{C} 26 & 48.3(5) & \mathrm{C} 27-\mathrm{O} 2-\mathrm{C} 24-\mathrm{C} 23 & 170.2(3) \\ \mathrm{C} 1-\mathrm{C} 3-\mathrm{C} 4-\mathrm{N} 1 & -176.3(3) & \mathrm{C} 27-\mathrm{O} 2-\mathrm{C} 24-\mathrm{C} 25 & -7.8(5) \\ \mathrm{C} 1-\mathrm{C} 3-\mathrm{C} 16-\mathrm{N} 5 & -176.3(3) & \mathrm{C} 2-\mathrm{C} 3-\mathrm{C} 4-\mathrm{N} 1 & 64.6(4) \\ \mathrm{C} 4-\mathrm{N} 1-\mathrm{C} 5-\mathrm{C} 6 & -172.0(3) & \mathrm{C} 2-\mathrm{C} 3-\mathrm{C} 16-\mathrm{N} 5 & -57.7(4)\end{array}$

Hydrogen-bond geometry $\left(A,{ }^{\circ}\right)$

\begin{tabular}{lllll}
\hline$D-\mathrm{H} \cdots A$ & $D-\mathrm{H}$ & $\mathrm{H} \cdots A$ & $D \cdots A$ & $D-\mathrm{H} \cdots A$ \\
\hline $\mathrm{C} 27-\mathrm{H} 27 A \cdots \mathrm{O} 1^{\mathrm{i}}$ & 0.96 & 2.60 & $3.517(4)$ & 161 \\
$\mathrm{C} 20-\mathrm{H} 20 B \cdots \mathrm{O} 2^{\mathrm{ii}}$ & 0.97 & 2.60 & $3.282(4)$ & 127 \\
$\mathrm{C} 19-\mathrm{H} 19 \cdots \mathrm{C} 28^{\mathrm{iii}}$ & 0.93 & 2.75 & $3.574(5)$ & 148 \\
$\mathrm{C} 19-\mathrm{H} 19 \cdots \mathrm{S} 1^{\mathrm{iii}}$ & 0.93 & 2.98 & $3.825(4)$ & 152 \\
$\mathrm{C} 17-\mathrm{H} 17 \cdots \mathrm{N} 10^{\mathrm{iii}}$ & 0.93 & 2.67 & $3.416(4)$ & 138 \\
$\mathrm{C} 17-\mathrm{H} 17 \cdots \mathrm{C} 29^{\mathrm{iii}}$ & 0.93 & 2.85 & $3.685(5)$ & 150 \\
$\mathrm{C} 16-\mathrm{H} 16 A \cdots \mathrm{C} 29^{\mathrm{iii}}$ & 0.97 & 2.73 & $3.667(5)$ & 163 \\
$\mathrm{C} 5-\mathrm{H} 5 \cdots \mathrm{N} 9^{\mathrm{iv}}$ & 0.93 & 2.67 & $3.590(5)$ & 173 \\
$\mathrm{C} 7-\mathrm{H} 7 \cdots \mathrm{N} 10^{\mathrm{iv}}$ & 0.93 & 2.75 & $3.614(5)$ & 156 \\
$\mathrm{C} 7-\mathrm{H} 7 \cdots \mathrm{C} 29^{\mathrm{iv}}$ & 0.93 & 2.49 & $3.400(5)$ & 166 \\
$\mathrm{C} 7-\mathrm{H} 7 \cdots \mathrm{S} 2^{\mathrm{iv}}$ & 0.93 & 2.99 & $3.752(5)$ & 140 \\
\hline
\end{tabular}

Symmetry codes: (i) $x+1 / 2, y-1,-z+1 / 2$; (ii) $-x+1, y+1 / 2,-z+1 / 2$; (iii) $-x+1,-y+1,-z+1$; (iv) $-x+1 / 2, y+1 / 2, z$. 